\title{
Mateusz Klonowski®
}

\section{History of Relating Logic. The Origin and Research Directions}

\begin{abstract}
In this paper we present the history of and the research directions in relating logic. For this purpose we will describe Epstein's Programme, which postulates accounting for the content of sentences in logical research. We will focus on analysing the content relationship and Epstein's logics that are based on it, which are special cases of relating logic. Moreover, the set-assignment semantics will be discussed. Next, the Torunian Programme of Relating Semantics will be presented; this programme explores the various non-logical relationships in logical research, including those which are content-related. We will present a general description of relating logic and semantics as well as the most prominent issues regarding the Torunian Programme, including some of its special cases and the results achieved to date.
\end{abstract}

Keywords: Epstein's Programme; relating logic; Torunian Programme of Relating Semantics

\section{Introduction}

By relating logic we mean any non-classical logic in which one accounts for the relation of sentences in terms of various connections: content relationship (analyticity, relevance, etc.), causality, temporal order, preference order, etc. [see Jarmużek, 2021; Jarmużek and Klonowski, 2021].

The first logics that might be considered to be relating logics were presented by Richard Epstein in 1976 at a seminar held at Victoria University of Wellington, New Zealand. They were given in response to a lecture on action theory given by Douglas Walton and to the problem of accounting for content relationship for an analysis of conditional sentences. 
Epstein defined his logics in a propositional language that included three connectives: negation, conjunction, and implication. While the negation and conjunction were interpreted classically, the interpretation of implication assumed the truth-condition of the material implication as well as a special relation between the antecedent and the consequent. Such a relation was to express the content relationship between the sentences [cf. Epstein, 1979; Epstein and Walton, 1979; Walton, 1979a,b]. Epstein decided to represent the content-related relationship between sentences using an at least reflexive binary relation defined on the set of propositional variables, extendable onto the set of formulae by means of certain special conditions. The idea aroused the interest not only of Walton but also of other seminar participants, including David Lewis, who noticed that Epstein's relation could be used to express the content relationship understood as overlapping of sentence content [see Epstein and Walton, 1979, p. 113; Epstein, 1979, pp. 156-158; Epstein, 1990, pp. 68-70]. Both Epstein and Walton have argued that many ideas related to the logics defined by Epstein were developed during the 1976 seminar [see Epstein and Walton, 1979, p. 113]. This includes not only the concept of content relationship, but also the proof of completeness for the systems being analysed, found together with Robert Goldblatt. ${ }^{1}$ The logics defined by Epstein were named relatedness logics. A relatedness logic consists of two systems: the logic $\mathbf{S}$, also known as the Symmetric Relatedness Logic, and the logic R, i.e., the Nonsymmetric Relatedness Logic, which is a sub-logic of $\mathbf{S}$.

In the 1980s Epstein presents further logics motivated by the analysis of content relationship, this time understood as the inclusion of sentence content. These are the so-called dependence logics, in particular the logic D, known as the Dependence Logic; the logic DD, known as the Dual Dependence Logic; and the logic Eq, known as the Logic of Equality of Contents [see Epstein, 1987]. A detailed description of these systems alongside the philosophical motivation is given in the monograph [Epstein,

1 From the description given by Epstein [1979, p. 156] it appears that Lewis introduced not only the notion of content relationship (understood as overlapping of sentence content) but also a content representing function, the so-called union set-assignment (see Section 2.3). Moreover, he at least suggested the relationships that might occur between the function he had introduced and Epstein's relation [see Epstein, 1979, pp. 156-158; Epstein, 1990, pp. 68-70]. All these notions and the relationships that exist between them will be described in further detail at a later stage (see Section 2.2-2.4). 
1990, pp. 115-143]. Apart from the above, Epstein [1990, pp. 142-143] also discusses the logic DPC, called the Classically-Dependent Logic. This logic can be viewed as a special case of dependence logic.

The monograph [Epstein, 1990] contains the most significant results on the logics defined by Epstein, including an analysis of intensional logics (e.g., modal logic) by means of a certain type of functional semantics (called set-assignment semantics), which was developed for the analysis of content relationship of sentences. This work is the cornerstone of the so-called Epstein Programme formulated by Krajewski [1991].

Although Epstein's systems may be considered to be examples of relating logic, the concept of relating logic was only presented in full as late as 24 years after the publication of Epstein's monograph. Tomasz Jarmużek and Bartosz Kaczkowski were the first to do so, with their paper [Jarmużek and Kaczkowski, 2014] describing the fragment of the smallest classical mono-relating logic (see Section 3.2). This logic is defined in a language that includes negation, conjunction, alternative, implication, and equivalence, which are interpreted classically, as well as conjunction and implication, which are interpreted in a relating manner, i.e., accounting for various non-logical relationships. It is worth noting that implication was the only relating connective in Epstein's logics. Although he believed that the relating interpretation of the disjunction also made sense and his main systems are functionally complete [see Epstein, 1979, p. 143; Epstein, 1990, pp. 71-72, 121, 77-80, 125].

The concept of relating interpretation of logical connectives was born out of discussions between Jarmużek and Kaczkowski when they met at a conference 13th International Workshop for Young Mathematicians. Logic and the Foundations of Mathematics held in Cracow in 2010. At the time neither of them was familiar with Epstein's works. Indeed, they only discovered Epstein's works from 1979 to 1990 when working on a presentation for a conference "Non-Classical Logic. Theory and Applications" held in Eódź in $2011 .^{2}$

Jarmużek and Kaczkowski's insight was in fact very simple; in nonextensional interpretations of connectives such as conjunction and implication one usually assumes that the component sentences are somehow interrelated. This relationship does not have to be content-related. We often consider sentences to be related because there is some relationship

2 The results presented at this conference are partly included in [Jarmużek and Kaczkowski, 2014]. 
that links the things, facts, states, etc. they refer to. The causal relationship and temporal order are two obvious examples. The fact that some sentences are somehow related to each other was formally represented via a binary relation defined on the set of formulae. This kind of relation could satisfy various conditions depending on the relationship which was to be accounted for in a given interpretation of a connective. Consequently, it became possible to define the foundations of relating semantics.

In 2020, Jarmużek proposed a general approach to relating semantics and gave examples of its applications in philosophical logic [see Jarmużek, 2021; Jarmużek and Klonowski, 2020, 2021]. In 2019, Jarmużek and Malinowski showed that relating semantics may be used to define connexive logics, thus establishing yet another chapter in the research on relating semantics [see Jarmużek and Malinowski, 2019a,b; Malinowski and Palczewski, 2021].

The ideas briefly presented above, including the analysis of Epstein's content relationship, were first described in the $\mathrm{PhD}$ thesis of Klonowski [2019], written under the supervision of Jarmużek. In this work, which accounts for the above mentioned results, we will attempt to pinpoint the key points of the Torunian Programme of Relating Semantics.

Section 2 will briefly describe the analysis of content relationship in Epstein's approach and the relatedness and dependence logics which stem from it, as well as the set-assignment semantics for selected modal logics. Section 3 will describe the Torunian Programme of Relating Semantics, as well as the general approach to relating logic/semantics; the main research directions will also be examined, including selected results of the Torun Logic Group. In Section 4, as a summary, we will compare Epstein's Programme to the Torunian Programme of Relating Semantics.

\section{Epstein's Programme}

Epstein's Programme was formulated by Krajewski [1991] based on the main results in [Epstein, 1990]. The key facets of this programme can be expressed as follows:

1. Main assumption: two properties of sentences are significant for logical research: the logical value and the content of sentences. 


\begin{tabular}{|l||l|l|}
\hline & Relational approach & Functional approach \\
\hline the content & $\begin{array}{l}\text { is determined by the content } \\
\text { relationship which is } \\
\text { a primitive notion }\end{array}$ & $\begin{array}{l}\text { is a (primitive) property } \\
\text { of a sentence }\end{array}$ \\
\hline formal tool & $\begin{array}{l}\text { extensions of relatedness } \\
\text { relation }\end{array}$ & union set-assignments \\
\hline $\begin{array}{l}\text { main } \\
\text { problem }\end{array}$ & $\begin{array}{l}\text { adequacy of the representation } \\
\text { of the content relationship }\end{array}$ & $\begin{array}{l}\text { properties of relations } \\
\text { defined by means of } \\
\text { union set-assignments }\end{array}$ \\
\hline
\end{tabular}

Table 1. Relational and functional approach to content

2. Main goals: introduction of new content relationship logics - known as Epstein's logics (goal 1) - and the semantic analysis of known non-classical logics based on the main assumption (goal 2).

3. Main assumption of Epstein's logics: a conditional sentence is true iff the following conditions are satisfied:

- the antecedent is false or the consequent is true

- the antecedent and the consequent are related because of their content.

As demonstrated in point 1, the programme postulates that in formal research two sentence properties should be accounted for: the logical value and the content of a sentence. Logical values are formally represented by numbers; for example, the classical values, i.e., true and false, are assigned 1 and 0, respectively. Because of these values, it is possible to analyse various relationships between sentences thanks to the transformation of the set of formulae into the set of numbers that represent logical values, i.e., thanks to the attribution of values.

With regards to the content of sentences, Epstein proposes two formal representation methods (cf. Table 1). The first might be called the relation based approach, and the second the function based approach. In the first case the generally understood relationship between sentences becomes the primitive notion and is represented by means of certain at least reflexive relations defined on the set of formulae. Such relations are an extension of the so-called relatedness relation (see Section 2.1). In this approach the content of a sentence is expressed as a set defined by this relation. In the second case, a sentence's content is also represented as a set, but as the output of functions that associated to any 
formula a subset of some fixed set, i.e., as the output of the so-called set-assignments (see Section 2.1). One can distinguish set-assignments, the so-called union set-assignments (see Section 2.3), which preserve the principle of content compositionality. In the first case the key tool for the analysis is the relatedness relation, whereas in the second set-assignment (and its special case union set-assignment) is employed.

Epstein's work introduces the following understandings of a content relationship:

- an overlap (a common part) of contents [Epstein, 1979, 1990]:

$A$ is content-related to $B$ iff the content of $A$ and the content of $B$ have something in common,

- an inclusion of content [Epstein, 1987, 1990]:

$A$ is content-related to $B$ iff the content of $B$ is included in the content of $A$, the content of $A$ is included in the content of $B$, the content of $A$ and the content of $B$ are the same.

These approaches have a relational and functional variant according to the two methods of representation with regards to sentential context (see table 1). We will describe these approaches based on the language of Epstein's logic, i.e., the following propositional language:

$$
\mathcal{L}_{\mathrm{E}}:=\langle\operatorname{Var},\{\neg, \wedge\},\{\rightarrow\},(,)\rangle,
$$

where $\operatorname{Var}:=\left\{p, q, r, p_{1}, q_{1}, r_{1}, \ldots\right\}$ is a set of propositional variables. The set of formulae of $\mathcal{L}_{\mathrm{E}}$ is defined in a standard way and is denoted by $\mathcal{L}_{\mathrm{E}}$. Following Epstein's example, we will use the following abbreviations for any $A, B \in \mathcal{L}_{\mathrm{E}}$ :

$$
\begin{aligned}
A \vee B & :=\neg(\neg A \wedge \neg B) \\
A \leftrightarrow B & :=(A \rightarrow B) \wedge(B \rightarrow A) .
\end{aligned}
$$

We will also use the following notation:

- the set of sub-formulae of the formula $A \in \mathcal{L}_{\mathrm{E}}$ is denoted by $\operatorname{sub}(A)$, - the set of all subsets of $X$ (the power set of $X$ ) is denoted by $\mathcal{P}(X)$,

- the set of propositional variables of the formula $A \in \mathcal{L}_{\mathrm{E}}$ is denoted by $\operatorname{var}(A)$,

- the set of ordered (non-ordered) pairs of elements from $\mathcal{L}_{\mathrm{E}}$ is denoted by $\mathcal{L}_{\mathrm{E}} \times \mathcal{L}_{\mathrm{E}}\left(\mathcal{L}_{\mathrm{E}}+\mathcal{L}_{\mathrm{E}}\right)$, 
- the complexity of formulae in $\mathcal{L}_{\mathrm{E}}$ is defined in a standard way using the function $\mathrm{c}: \mathcal{L}_{\mathrm{E}} \longrightarrow \mathbb{N}$ such that $\mathrm{c}(A):=0$, for any $A \in \operatorname{Var}$.

\subsection{Relatedness relation and set-assignment}

At this point, we will move on to the description of the relatedness relation. As mentioned in the introduction, the starting point for Epstein's work from 1976 (presented in 1979) was an analysis of how conditional sentences are interpreted, in particular the shortcomings of interpretation of such sentences as a material implication. Known examples of the so-called material implication paradoxes indicate that the component sentences of true conditional sentences are usually somehow interrelated.

What we're really concerned with is making $p \rightarrow q$ true if and only if $p$ is related to $q$, and we don't have $p$ true and $q$ false. But what do we mean by 'related'? What does it mean to say that ' $p$ is related to $q$ '? Very simply, we've decided to take 'relatedness' as a primitive notion. For $p, q, \ldots$ can be taken to be anything one wants: sentences in English, or descriptions of events, or points in space-time, or mathematical statements, or $[\ldots]$.

For $p \rightarrow q$ we're only concerned with whether $p$ is related to $q$. So the idea is to take relatedness to be a binary relation, $r(p, q)$. That is, depending on whatever we are modelling we assume that we're given a predicate $r(p, q)$ that holds if $p$ is related to $q$, and fails if they are unrelated.

[Epstein, 1979, p. 139]

As we can see, the relatedness of sentences is to be the primitive notion; we do not specify which kind of relationship it represents, thus leaving various possibilities open. We adopt a general perspective assuming that the propositional variables can represent whatever we want. However, it seems that Epstein narrows this general perspective:

So far we've required only one property of relatedness: it is binary. In Section VI we consider ternary and indeed $n$-ary relatedness notions $[\ldots]$.

Do we need to assume any other properties? Several come to mind: symmetry, transitivity, etc. But these are all specific attributes of relatedness that may, or may not, apply to specific studies. For our general study, we need only one more property: reflexivity. That is, every proposition is related to itself, $r(p, p)$. [Epstein, 1979, p. 140] 
If reflexivity is a generally accepted assumption, can the relatedness relation allow us to analyse those relationships which are irreflexive or simply not reflexive?

If we're trying to model a natural language then this seems reasonable: every sentence is 'content related' to itself. Similarly, if we're concerned with modelling actions, then if we think of $r(p, q)$ as ' $p$ is close to $q$ in space and time', a necessary prerequisite for $p$ to 'cause' $q$, then again reflexivity is natural. (Note well that we do not believe that $r(p, q)$ can model 'causation' by itself; we don't believe that $p \rightarrow q$ should be read as ' $p$ causes $q$ '; rather $p \rightarrow q$ should be a prerequisite for ' $p$ causes $q$ '.) $[\ldots]$

Now you are free to require that relatedness satisfy whatever properties you wish when you want to use this system. However, the only property that is essential to make the system make sense, and must always be there, is reflexivity.

[Epstein, 1979, p. 140-141]

Consequently, it is possible to state that the notion of relatedness facilitates an analysis of various relationships provided that these relationships at least connect any object from the domain with itself. Epstein's approach using the relatedness relation may be summarised in the following points:

1. Relatedness of sentences is a primitive notion.

2. Relatedness of sentences is understood to be a relationship between objects denoted by propositional variables.

3. Relatedness of sentences may be represented by a reflexive relation defined on the set of propositional variables, i.e., by the relatedness relation.

4. Using various conditions, the relatedness relation may be extended onto the entire set of formulae.

5. By requesting further conditions for the relatedness relation and its extensions onto the entire set of formulae, we make our understanding of the relatedness of sentences more specific.

The next area of exploration is the description of set-assignment, i.e., the main tool for the analysis of content and content relationship introduced by Epstein. This is a mapping $s: F \longrightarrow \mathcal{P}(S)$, in which $F$ is a set of formulae and $S$ is a set (non-empty unless stated otherwise). In the following section, we will focus on the set-assignments for which the domain is $\mathcal{L}_{\mathrm{E}}$. By using special set-assignments $s: \mathcal{L}_{\mathrm{E}} \longrightarrow \mathcal{P}(S)$, satisfying the principle of content compositionality, Epstein [1979, 1990] 
assigns sets to formulae which represent sentences. These sets represent the content of respective sentences. Such set-assignments will be described further on (see Section 2.3).

Let us note that an example of set-assignment is provided by the classical operation of logical consequence defined over $\mathcal{L}_{\mathrm{E}}$ (which will be denoted by $\left.\mathrm{Cn}_{\mathcal{L}_{\mathrm{E}}}: \mathcal{L}_{\mathrm{E}} \longrightarrow \mathcal{P}\left(\mathcal{L}_{\mathrm{E}}\right)\right)$. By using such a function, the content of a sentence $A$ may be considered identical with the set of values of $\mathrm{Cn}_{\mathcal{L}_{\mathrm{E}}}$ on $A$. However, the logical consequence may also be expressed by using the classical relation of logical consequence, i.e., a certain relation $\models_{\mathcal{L}_{\mathrm{E}}} \subseteq \mathcal{L}_{\mathrm{E}} \times \mathcal{L}_{\mathrm{E}} \cdot{ }^{3}$ Obviously, the restriction of such a relation to propositional variables is an example of a relatedness relation. In this case, the content of sentence $A$ may be equated with the set of sentences which are in relation $\models_{\mathcal{L}_{\mathrm{E}}}$ with $A$. Because of properties such as reflexivity and transitivity, as well as the mutual definability of $\mathrm{Cn}_{\mathcal{L}_{\mathrm{E}}}$ and $\models_{\mathcal{L}_{\mathrm{E}}}$, the following is true:

$$
A \models \mathcal{L}_{\mathrm{E}} B \text { iff } \mathrm{Cn}_{\mathcal{L}_{\mathrm{E}}}(B) \subseteq \mathrm{C}_{\mathcal{L}_{\mathrm{E}}}(A),
$$

for any $A, B \in \mathcal{L}_{\mathrm{E}}$. Thus, given a representation of the sentence content, one can express the content relationship understood as inclusion (B1) both in the relational and functional approach. Both a set-assignment and an extension of relatedness relation can serve as the starting point for analysis of such a content relationship. In the former, based on a given set-assignment one can produce a certain extension of relatedness relation, while in the latter one can produce a certain set-assignment based on a given extension of relatedness relation.

The next area of discussion is the analysis of content relationship using certain extensions of the relatedness relation as proposed by Epstein; after that, we will consider an approach to content relationship using setassignments which satisfy the principle of content compositionality.

\subsection{Extensions of the relatedness relation}

Aside from reflexivity, Epstein [1979, p. 141, 1990, pp. 65-67] proposes other relational conditions which in his opinion are natural in the context of analysis of the broadly understood content relationship. As a consequence, any relation which satisfies such conditions will be called a relatedness relation of content.

${ }^{3}$ Usually, the domain of the consequence relation is the power set of the set of formulae; however, in our example the domain is restricted to the set of formulae. 
By a relatedness relation of content (for short: an $r$-relation) we will mean a relation $R \subseteq \mathcal{L}_{\mathrm{E}} \times \mathcal{L}_{\mathrm{E}}$ which satisfies the following conditions:

$$
\begin{aligned}
& \forall_{A \in \mathcal{L}_{\mathrm{E}}} R(A, A), \\
& \forall_{A, B \in \mathcal{L}_{\mathrm{E}}}(R(A, \neg B) \text { iff } R(A, B)), \\
& \forall_{A, B, C \in \mathcal{L}_{\mathrm{E}}}(R(A, B \wedge C) \text { iff } R(A, B \rightarrow C)), \\
& \forall_{A, B, C \in \mathcal{L}_{\mathrm{E}}}(R(A, B \wedge C) \text { iff }(R(A, B) \text { or } R(A, C))), \\
& \forall_{A, B \in \mathcal{L}_{\mathrm{E}}}(R(\neg A, B) \text { iff } R(A, B)), \\
& \forall_{A, B, C \in \mathcal{L}_{\mathrm{E}}}(R(A \wedge B, C) \text { iff } R(A \rightarrow B, C)), \\
& \forall_{A, B, C \in \mathcal{L}_{\mathrm{E}}}(R(A \wedge B, C) \text { iff }(R(A, C) \text { or } R(B, C))) .
\end{aligned}
$$

The following studies selected r-relation properties. To begin, it should be noted that formulae are in the r-relation when some of the propositional variables from these formulae are in this r-relation. Obviously, a restriction of an arbitrary r-relation to Var is a relatedness relation.

FACT 2.1 (Epstein, 1990, p. 67, Lemma 1). Let $R$ be an r-relation. Then, for any $A, B \in \mathcal{L}_{E}, R(A, B)$ iff $\exists_{a \in \operatorname{var}(A)} \exists_{b \in \operatorname{var}(B)} R(a, b)$.

The equivalence stated in Fact 2.1 also holds for relations which are not reflexive yet satisfy all the remaining conditions of an r-relation. With reference to the equivalence from Fact 2.1, there is a simple method to extend any relatedness relation to an r-relation.

FACT 2.2. Let $Q$ be a relatedness relation and $R \subseteq \mathcal{L}_{E} \times \mathcal{L}_{E}$ be an extension of $Q$ onto $\mathcal{L}_{E}$, defined for any $A, B \in \mathcal{L}_{E}$ as follows: $R(A, B)$ iff $\exists_{a \in \operatorname{var}(A)} \exists_{b \in \operatorname{var}(B)} Q(a, b)$. The $R$ is an r-relation.

Proof. For $\left(\mathrm{r}_{1}\right)$. By the definition of relatedness relation, for any $A \in \operatorname{var}(A), Q(A, A)$. Thus, $\exists_{a \in \operatorname{var}(A)} \exists_{b \in \operatorname{var}(A)} Q(a, b)$. Consequently, $R(A, A)$.

For $\left(\mathrm{r}_{2}\right)$. We have: $R(A, \neg B)$, by the definition of $R$, iff $\exists_{a \in \operatorname{var}(A)}$ $\exists_{b \in \operatorname{var}(\neg B)} Q(a, b)$, by the equality $\operatorname{var}(\neg B)=\operatorname{var}(B)$, iff $\exists_{a \in \operatorname{var}(A)} \exists_{b \in \operatorname{var}(B)}$ $Q(a, b)$, by the definition of $R$, iff $R(A, B)$.

For $\left(\mathrm{r}_{3}\right)$. We have: $R(A, B \wedge C)$, by the definition of $R$, iff $\exists_{a \in \operatorname{var}(A)}$ $\exists_{b \in \operatorname{var}(B \wedge C)} Q(a, b)$, by the equality $\operatorname{var}(B \wedge C)=\operatorname{var}(B) \cup \operatorname{var}(C)=$ $\operatorname{var}(B \rightarrow C)$, iff $\exists_{a \in \operatorname{var}(A)} \exists_{b \in \operatorname{var}(B \rightarrow C)} Q(a, b)$, by the definition of $R$, iff $R(A, B \rightarrow C)$.

For $\left(\mathrm{r}_{4}\right)$. We have: $R(A, B \wedge C)$, by the definition of $R$, iff $\exists_{a \in \operatorname{var}(A)}$ $\exists_{b \in \operatorname{var}(B \wedge C)} Q(a, b)$, by the equality $\operatorname{var}(B \wedge C)=\operatorname{var}(B) \cup \operatorname{var}(C)$, iff 
$\exists_{a \in \operatorname{var}(A)} \exists_{b \in \operatorname{var}(B) \cup \operatorname{var}(C)} Q(a, b)$, by the definition of the union and the laws of classical logic, iff $\left(\exists_{a \in \operatorname{var}(A)} \exists_{b \in \operatorname{var}(B)} Q(a, b)\right.$ or $\exists_{a \in \operatorname{var}(A)} \exists_{b \in \operatorname{var}(C)}$ $Q(a, b))$, by the definition of $R$, iff $(R(A, B)$ or $R(A, C))$.

For $\left(\mathrm{r}_{5}\right),\left(\mathrm{r}_{6}\right)$, and $\left(\mathrm{r}_{7}\right)$, respectively, we make a similar argument as for $\left(\mathrm{r}_{2}\right)\left(\mathrm{r}_{3}\right)$, and $\left(\mathrm{r}_{4}\right)$.

In order to express the content relationship between sentences, understood as the common part (overlap) of the sentence content (A), Epstein [1990, pp. 61-68] refers to a symmetric r-relation (for short: an sr-relation), i.e., an r-relation that is symmetric. A relation $R \subseteq \mathcal{L}_{\mathrm{E}} \times \mathcal{L}_{\mathrm{E}}$ is symmetric iff the following condition holds:

$$
\forall_{A, B \in \mathcal{L}_{\mathrm{E}}}(R(A, B) \Rightarrow R(B, A)) .
$$

The following fact defines the minimal set of conditions which must be met by an sr-relation:

FACT 2.3. $R$ is an sr-relation iff $R$ satisfies conditions $\left(\mathrm{r}_{1}\right)-\left(\mathrm{r}_{4}\right)$ and $\left(\mathrm{r}_{8}\right)$. Proof. " $\Rightarrow$ " Obvious.

" $\Leftarrow$ " Let $R$ satisfy conditions $\left(\mathrm{r}_{1}\right)-\left(\mathrm{r}_{4}\right)$ and $\left(\mathrm{r}_{8}\right)$. Then, for any $A, B \in \mathcal{L}_{\mathrm{E}}: R(\neg A, B)$, by condition $\left(\mathrm{r}_{8}\right)$, iff $R(B, \neg A)$, by condition $\left(\mathrm{r}_{2}\right)$, iff $R(B, A)$, and by condition $\left(\mathrm{r}_{8}\right)$, iff $R(A, B)$. Therefore, $R$ satisfies conditions $\left(\mathrm{r}_{5}\right)$. The proof that $R$ satisfies conditions $\left(\mathrm{r}_{6}\right)$ and $\left(\mathrm{r}_{7}\right)$ is similar; one only needs to refer to conditions $\left(\mathrm{r}_{3}\right),\left(\mathrm{r}_{4}\right)$, and $\left(\mathrm{r}_{8}\right)$.

In order to prove that there is a way to represent content relationship (A) by means of sr-relations we will define a certain set-assignment. ${ }^{4}$ Let $R$ be an sr-relation. The $\mathrm{s}_{R}: \mathcal{L}_{\mathrm{E}} \longrightarrow \mathcal{P}\left(\mathcal{L}_{\mathrm{E}}+\mathcal{L}_{\mathrm{E}}\right)$ function is defined as follows:

$$
\mathrm{s}_{R}(A):=\left\{\{A, B\} \in \mathcal{L}_{\mathrm{E}}+\mathcal{L}_{\mathrm{E}}: R(A, B)\right\} .
$$

The following fact holds:

FACT 2.4 (Epstein, 1990, p. 68, Lemma 2). Let $R$ be an sr-relation. Then, for any $A, B \in \mathcal{L}_{E}, R(A, B)$ iff $\mathrm{s}_{R}(A) \cap \mathrm{s}_{R}(B) \neq \emptyset$.

A similar analysis can be performed for an r-relation, which does not have to be symmetric. To this end we define two set-assignments for the same r-relation. ${ }^{5}$

4 Epstein [1990, p. 68] calls this function subject matter set-assignment associated with $R$, where $R$ is an sr-relation.

5 This approach has been proposed by Krajewski [1991, pp. 19-20]. 
Let $R$ be an r-relation. The $\mathrm{s}_{R}, \mathrm{~s} 2_{R}: \mathcal{L}_{\mathrm{E}} \longrightarrow \mathcal{P}\left(\mathcal{L}_{\mathrm{E}} \times \mathcal{L}_{\mathrm{E}}\right)$ functions are defined as follows:

$$
\begin{aligned}
& \mathrm{s} 1_{R}(A):=\left\{\langle A, B\rangle \in \mathcal{L}_{\mathrm{E}} \times \mathcal{L}_{\mathrm{E}}: R(A, B)\right\}, \\
& \mathrm{s} 2_{R}(A):=\left\{\langle B, A\rangle \in \mathcal{L}_{\mathrm{E}} \times \mathcal{L}_{\mathrm{E}}: R(B, A)\right\} .
\end{aligned}
$$

Similar to an sr-relation, a relation which is an r-relation but is not symmetric allows us to express content relationship understood as the common part of the sentence content:

FACT 2.5. Let $R$ be an r-relation. Then, for any $A, B \in \mathcal{L}_{E}, R(A, B)$ iff $\mathrm{s}_{R}(A) \cap \mathrm{s} 2_{R}(B) \neq \emptyset$.

Proof. " $\Rightarrow$ " Assume that $R(A, B)$. Using the definition of $\mathrm{s} 1_{R}, \mathrm{~s}_{2}$, $\langle A, B\rangle \in \mathrm{s} 1_{R}(A)$, and $\langle A, B\rangle \in \mathrm{s} 2_{R}(B)$. So $\mathrm{s}_{R}(A) \cap \mathrm{s} 2_{R}(B) \neq \emptyset$.

" $\Leftarrow$ " Assume that $\mathrm{s}_{R}(A) \cap \mathrm{s} 2_{R}(B) \neq \emptyset$. Therefore, $X \in \mathrm{s} 1_{R}(A)$ and $X \in \mathrm{s} 2_{R}(B)$, for some $X \in \mathcal{L}_{\mathrm{E}} \times \mathcal{L}_{\mathrm{E}}$. By the definition of $\mathrm{s} 1_{R}, \mathrm{~s}_{R}$, $X=\langle A, C\rangle$ and $X=\langle D, B\rangle$. Thus, $A=D$ and $B=C$. Hence $X=\langle A, B\rangle$. Consequently, using the definition of $\mathrm{s} 1_{R}, \mathrm{~s} 2_{R}, R(A, B) . \dashv$

From here we can move onto the representation of content relationship understood as the inclusion of sentence content in the sense of (B1)-(B3). Following Epstein's [1990] example, one can define a content dependence relation, a dual content dependence relation and a content equality relation. It is worth mentioning that Epstein [1990, p. 122, Lemma 1; p. 137, Theorem 9] defines the content dependence relation and the content equality relation indirectly by using union setassignment (see Section 2.3). In turn, the dual content dependence relation is understood as the converse of the content dependence relation in its original formulation. In the proposed description, we will begin with the relational approach before employing the functional approach on a subsequent basis.

By a content dependence relation (for short: a d-relation), we mean a relation $R \subseteq \mathcal{L}_{\mathrm{E}} \times \mathcal{L}_{\mathrm{E}}$ which satisfies $\left(\mathrm{r}_{1}\right)$ (reflexivity) as well as the following conditions:

$$
\begin{aligned}
& \forall_{A, B, C \in \mathcal{L}_{\mathrm{E}}}((R(A, B) \text { and } R(B, C)) \Rightarrow R(A, C)), \\
& \forall_{A, B, C \in \mathcal{L}_{\mathrm{E}}}(R(A, B \wedge C) \text { iff }(R(A, B) \text { and } R(A, C)), \\
& \forall_{A, B, C \in \mathcal{L}_{\mathrm{E}}}(R(A, B \rightarrow C) \text { iff }(R(A, B) \text { and } R(A, C))), \\
& \forall_{A \in \mathcal{L}_{\mathrm{E}}} R(\neg A, A), \\
& \forall_{A \in \mathcal{L}_{\mathrm{E}}} R(A, \neg A) .
\end{aligned}
$$


The content dependence relation allows the representation of a relationship understood as the inclusion of sentence content (B1).

Note that if an r-relation satisfied condition $\left(\mathrm{d}_{1}\right)$, i.e., if it was a transitive r-relation, then given conditions $\left(\mathrm{r}_{1}\right),\left(\mathrm{r}_{4}\right)$, and $\left(\mathrm{r}_{7}\right)$ it would be a universal relation [cf. Epstein, 1990, p. 76]. By $\left(\mathrm{r}_{1}\right), R(A, A)$ and $R(B$, $B)$. By $\left(\mathrm{r}_{4}\right)$ and $\left(\mathrm{r}_{7}\right),(R(A, A)$ or $R(A, B)) \Rightarrow R(A, A \wedge B)$ and $(R(A, B)$ or $R(B, B)) \Rightarrow R(A \wedge B, B)$ respectively. Hence, $R(A, A \wedge B)$ and $R(A$ $\wedge B, B)$. By transitivity $\left(\mathrm{d}_{1}\right), R(A, B)$.

The following fact shows some similarities between a d-relation and an r-relation, and also shows that condition $\left(\mathrm{r}_{1}\right)$ in the definition of a d-relation can be skipped.

FACT 2.6. Let $R \subseteq \mathcal{L}_{E} \times \mathcal{L}_{E}$. Then:

1. if $R$ satisfies conditions $\left(\mathrm{d}_{1}\right),\left(\mathrm{d}_{4}\right)$, and $\left(\mathrm{d}_{5}\right)$, then it also satisfies conditions $\left(\mathrm{r}_{1}\right),\left(\mathrm{r}_{2}\right)$, and $\left(\mathrm{r}_{5}\right)$;

2. if $R$ satisfies conditions $\left(\mathrm{r}_{1}\right),\left(\mathrm{r}_{2}\right)$, and $\left(\mathrm{r}_{5}\right)$, then it also satisfies conditions $\left(\mathrm{d}_{4}\right)$ and $\left(\mathrm{d}_{5}\right)$.

Proof. For 1. By $\left(\mathrm{d}_{1}\right),(R(A, \neg A)$ and $R(\neg A, A)) \Rightarrow R(A, A)$. Hence, by $\left(\mathrm{d}_{5}\right)$ and $\left(\mathrm{d}_{4}\right), R(A, A)$. By $\left(\mathrm{d}_{1}\right),(R(A, B)$ and $R(B, \neg B)) \Rightarrow R(A$, $\neg B)$. Thus $R(B, \neg B) \Rightarrow(R(A, B) \Rightarrow R(A, \neg B))$. Consequently, by $\left(\mathrm{d}_{5}\right), R(A, B) \Rightarrow R(A, \neg B)$ and by $\left(\mathrm{d}_{1}\right),(R(A, \neg B)$ and $R(\neg B, B)) \Rightarrow$ $R(A, B)$. Hence $R(\neg B, B) \Rightarrow(R(A, \neg B) \Rightarrow R(A, B))$. Thus, by $\left(\mathrm{d}_{4}\right)$, $R(A, \neg B) \Rightarrow R(A, B)$. Consequently, $R(A, \neg B)$ iff $R(A, B)$. A similar argument proves $\left(\mathrm{r}_{5}\right)$.

For 2. By $\left(\mathrm{r}_{2}\right), R(A, A)$ iff $R(A, \neg A)$. Thus, by $\left(\mathrm{r}_{1}\right), R(A, \neg A)$. $\mathrm{A}$ similar argument proves $\left(\mathrm{d}_{5}\right)$.

Unlike an r-relation, a d-relation cannot by characterised by the relatedness relation and quantifying propositional variables (cf. Fact 2.1), although its restriction to propositional variables is obviously a relatedness relation. However, the following fact holds:

FACT 2.7 (cf. Epstein, 1990, p. 123, Lemma 2). Let $R$ be a d-relation. Then, for any $A, B \in \mathcal{L}_{E}, R(A, B)$ iff $\forall_{a \in \operatorname{var}(B)} R(A, a)$.

Proof. Let $A \in \mathcal{L}_{\mathrm{E}}$. The proof will be by induction on the complexity of the $\mathcal{L}_{\mathrm{E}}$ formulae.

Initial step. Let $B \in \mathcal{L}_{\mathrm{E}}$ and $\mathrm{c}(B)=0$. Consequently, $\operatorname{var}(B)=\{B\}$. It follows that $R(A, B)$ iff $\forall_{a \in \operatorname{var}(B)} R(A, a)$. 
Inductive hypothesis. Let $n \in \mathbb{N}$. Assume that for any $B \in \mathcal{L}_{\mathrm{E}}$, if $\mathrm{c}(B) \leqslant n$, then $\left(R(A, B)\right.$ iff $\left.\forall_{a \in \operatorname{var}(B)} R(A, a)\right)$.

Inductive step. Let $B \in \mathcal{L}_{\mathrm{E}}$ and $\mathrm{c}(B)=n+1$. Consider the following cases of formula construction in $\mathcal{L}_{\mathrm{E}}$ :

Let $B=\neg C$. Then: $R(A, B)$ iff $R(A, \neg C)$, given Fact 2.6.2, iff $R(A, C)$, by the inductive hypothesis, iff $\forall_{a \in \operatorname{var}(C)} R(A, a)$, by the equality $\operatorname{var}(\neg C)=\operatorname{var}(C)$, iff $\forall_{a \in \operatorname{var}(\neg C)} R(A, a)$.

Let $B=C * D$, where $* \in\{\wedge, \rightarrow\}$. Then: $R(A, B)$ iff $R(A, C * D)$, by $\left(\mathrm{d}_{2}\right)$ and $\left(\mathrm{d}_{3}\right)$, iff $(R(A, C)$ and $R(A, D))$, by the inductive hypothesis, iff $\left(\forall_{a \in \operatorname{var}(C)} R(A, a)\right.$ and $\left.\forall_{a \in \operatorname{var}(D)} R(A, a)\right)$, by the laws of classical logic, iff $\forall a((a \in \operatorname{var}(C) \Rightarrow R(A, a))$ and $(a \in \operatorname{var}(D) \Rightarrow R(A, a)))$, by the laws of classical logic, iff $\forall a((a \in \operatorname{var}(C)$ or $a \in \operatorname{var}(D)) \Rightarrow R(A, a))$, by the definition of the union, iff $\forall a(a \in \operatorname{var}(C) \cup \operatorname{var}(D) \Rightarrow R(A, a))$, by equality $\operatorname{var}(C * D)=\operatorname{var}(C) \cup \operatorname{var}(D)$, iff $\forall_{a \in \operatorname{var}(C * D)} R(A, a)$.

We can prove that a relation that is reflexive, transitive, and satisfies the condition stated in Fact 2.7 is a d-relation.

FACT 2.8 (cf. Epstein, 1990, p. 123, Lemma 2). Let $R \subseteq \mathcal{L}_{E} \times \mathcal{L}_{E}$ satisfy conditions $\left(\mathrm{r}_{1}\right),\left(\mathrm{d}_{1}\right)$ and $(\dagger)$ : for any $A, B \in \mathcal{L}_{E}, R(A, B)$ iff for any $a \in \operatorname{var}(B)$ we have $R(A, a)$. Then $R$ is a d-relation.

Proof. For $\left(\mathrm{d}_{2}\right),\left(\mathrm{d}_{3}\right)$. Let $* \in\{\wedge, \rightarrow\}$. We have: $R(A, B * C)$, by $(\dagger)$, iff $\forall_{a \in \operatorname{var}(B * C)} R(A, a)$, by equality $\operatorname{var}(B * C)=\operatorname{var}(B) \cup \operatorname{var}(C)$, iff $\forall_{a \in \operatorname{var}(B) \cup \operatorname{var}(C)} R(A, a)$, by the sum definition and the laws of classical logic, iff $\forall a((a \in \operatorname{var}(B)$ or $a \in \operatorname{var}(C)) \Rightarrow R(A, a))$, by the laws of classical logic, iff $\forall a((a \in \operatorname{var}(B) \Rightarrow R(A, a))$ and $(a \in \operatorname{var}(C) \Rightarrow R(A, a)))$, by the laws of classical logic, iff $\left(\forall_{a \in \operatorname{var}(B)} R(A, a)\right.$ and $\left.\forall_{a \in \operatorname{var}(C)} R(A, a)\right)$, by $(\dagger)$, iff $(R(A, B)$ and $R(A, C))$.

For $\left(\mathrm{d}_{4}\right)$. We have: $R(\neg A, \neg A)$, by $(\dagger)$, iff $\forall_{a \in \operatorname{var}(\neg A)} R(\neg A, a)$, by equality $\operatorname{var}(\neg A)=\operatorname{var}(A)$, iff $\forall_{a \in \operatorname{var}(A)} R(\neg A, a)$, by $(\dagger)$, iff $R(\neg A, A)$. By $\left(\mathrm{r}_{1}\right) R(\neg A, \neg A)$, so $R(\neg A, A)$.

For $\left(\mathrm{d}_{5}\right)$ we make a similar argument as for $\left(\mathrm{d}_{4}\right)$.

In order to show that a d-relation allows for the expression of content relationship understood as inclusion (B1), it is necessary to define an appropriate function which is an example of set-assignment. Let $R$ be a d-relation. The $t_{R}: \mathcal{L}_{\mathrm{E}} \longrightarrow \mathcal{P}\left(\mathcal{L}_{\mathrm{E}}\right)$ function is defined as follows:

$$
\mathrm{t}_{R}(A):=\left\{B \in \mathcal{L}_{\mathrm{E}}: R(B, A)\right\} .
$$


The $\overline{\mathrm{t}}_{R}: \mathcal{L}_{\mathrm{E}} \longrightarrow \mathcal{P}\left(\mathcal{L}_{\mathrm{E}}\right)$ function is defined as follows:

$$
\overline{\mathrm{t}}_{R}(A):=\mathcal{L}_{\mathrm{E}} \backslash \mathrm{t}_{R}(A) .
$$

The definitions of $t_{R}$ and $\bar{t}_{R}$ are given by Epstein [1990, p. 122, Lemma 1]. The following fact holds:

FACT 2.9 (Epstein, 1990, p. 122, Lemma 1). Let $R$ be a d-relation. Then, for any $A, B \in \mathcal{L}_{E}, R(A, B)$ iff $\overline{\mathrm{t}}_{R}(B) \subseteq \overline{\mathrm{t}}_{R}(A)$.

From here we can define the dual content dependence relation. In this study a dual content dependence relation (for short: a dd-relation) refers to a relation $R \subseteq \mathcal{L}_{\mathrm{E}} \times \mathcal{L}_{\mathrm{E}}$ which satisfies the following conditions: $\left(\mathrm{r}_{1}\right)$ (reflexivity), $\left(\mathrm{d}_{1}\right)$ (transitivity), $\left(\mathrm{d}_{4}\right)$, and $\left(\mathrm{d}_{5}\right)$ (dependence between the formula and its negation). It also needs to satisfy the following further conditions:

$$
\begin{aligned}
& \forall_{A, B, C \in \mathcal{L}_{\mathrm{E}}}(R(A \wedge B, C) \text { iff }(R(A, C) \text { and } R(B, C))), \\
& \forall_{A, B, C \in \mathcal{L}_{\mathrm{E}}}(R(A \rightarrow B, C) \text { iff }(R(A, C) \text { and } R(B, C))) .
\end{aligned}
$$

The dd-relation is designed to allow for the representation of relatedness understood as the inclusion of sentence content (B2). ${ }^{6}$

It is easy to see that the converse of a d-relation is a dd-relation. Consequently, for a given dd-relation we can define the counterparts of Facts 2.7 and 2.8. To do so, it suffices to modify the condition stated in Fact 2.7, so also condition $(\dagger)$ in Fact 2.8, as follows ( $\ddagger$ ): for any $A, B \in \mathcal{L}_{\mathrm{E}}, R(A, B)$ iff for any $a \in \operatorname{var}(A)$ we have $R(a, B)$.

As can be seen, $(\ddagger)$ differs from $(\dagger)$ only in the order of quantification: instead of quantifying over the propositional variables of the second argument of the relation, we now quantify over the propositional variables of the first argument.

By modifying the definitions of functions $t_{R}$ and $\bar{t}_{R}$, we obtain functions which preserve an appropriate relationship with respect to the dd-relation. Functions $\mathrm{u}_{R}: \mathcal{L}_{\mathrm{E}} \longrightarrow \mathcal{P}\left(\mathcal{L}_{\mathrm{E}}\right)$ and $\overline{\mathrm{u}}_{R}: \mathcal{L}_{\mathrm{E}} \longrightarrow \mathcal{P}\left(\mathcal{L}_{\mathrm{E}}\right)$ are defined as follows:

$$
\begin{aligned}
& \mathrm{u}_{R}(A):=\left\{B \in \mathcal{L}_{\mathrm{E}}: R(A, B)\right\}, \\
& \overline{\mathrm{u}}_{R}(A):=\mathcal{L}_{\mathrm{E}} \backslash \mathrm{u}_{R}(A) .
\end{aligned}
$$

${ }^{6}$ It is important to note that Epstein does not introduce the notion of dual content dependence relation. Nonetheless, he does define a dual version of logic defined by means of d-relations by modifying the truth-condition for an implication. This logic may be defined directly using dd-relations (see Section 2.5). 
The following fact holds:

FACT 2.10. Let $R$ be a dd-relation. Then, for any $A, B \in \mathcal{L}_{E}, R(A, B)$ iff $\overline{\mathrm{u}}_{R}(A) \subseteq \overline{\mathrm{u}}_{R}(B)$.

Proof. " $\Rightarrow$ " Assume that $R(A, B)$ and that $C \in \overline{\mathrm{u}}_{R}(A)$. If $C \notin \overline{\mathrm{u}}_{R}(B)$, then $C \in \mathrm{u}_{R}(B)$. Thus, $R(B, C)$. Hence, by $\left(\mathrm{d}_{1}\right), R(A, C)$.

" $\Leftarrow$ " Assume that $\overline{\mathrm{u}}_{R}(A) \subseteq \overline{\mathrm{u}}_{R}(B)$. Thus, by $\left(\mathrm{r}_{1}\right), B \notin \overline{\mathrm{u}}_{R}(A)$. Consequently, $R(A, B)$.

The final example of an extension of the relatedness relation analysed by Epstein is the content equality relation. Here, the term content equality relation (for short: an eq-relation) refers to a relation $R \subseteq \mathcal{L}_{\mathrm{E}} \times \mathcal{L}_{\mathrm{E}}$ which satisfies the following conditions: $\left(\mathrm{r}_{1}\right)$ (reflexivity), $\left(\mathrm{r}_{8}\right)$ (symmetry), $\left(\mathrm{d}_{1}\right)$ (transitivity), and $\left(\mathrm{d}_{5}\right)$ (dependence between the formula and its negation). It is also required to satisfy several further conditions:

$$
\begin{aligned}
& \forall_{A, B, C \in \mathcal{L}_{\mathrm{E}}}(R(A, B) \Rightarrow R(A \wedge C, B \wedge C)), \\
& \forall A, B \in \mathcal{L}_{\mathrm{E}} R(A \wedge B, B \wedge A), \\
& \forall A, B \in \mathcal{L}_{\mathrm{E}} R(A \rightarrow B, A \wedge B), \\
& \forall A, B, C \in \mathcal{L}_{\mathrm{E}} R(A \wedge(B \wedge C),(A \wedge B) \wedge C), \\
& \forall_{A, B, C, D \in \mathcal{L}_{\mathrm{E}}}((R(A, B \wedge C) \text { and } R(B, A \wedge D)) \Rightarrow R(A, B)) \text {, }
\end{aligned}
$$

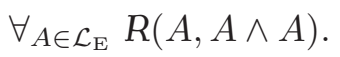

The content equality relation is designed to allow the representation of a relationship understood as sentence content equality (B3). ${ }^{7}$ In order to prove that this approach is correct, Epstein defines an auxiliary drelation using an eq-relation.

Let $R$ to be an eq-relation. The relation $\mathrm{R}_{R} \subseteq \mathcal{L}_{\mathrm{E}} \times \mathcal{L}_{\mathrm{E}}$ is defined for any $A, B \in \mathcal{L}_{\mathrm{E}}$ as follows:

$$
\mathrm{R}_{R}(A, B) \text { iff } \exists_{C \in \mathcal{L}_{\mathrm{E}}}(B \in \operatorname{sub}(C) \text { and } R(A, C)) .
$$

The following fact therefore holds:

FACT 2.11 (Epstein, 1990, p. 138, Lemma 11). If $R$ is an eq-relation, then $\mathrm{R}_{R}$ is a d-relation.

7 Similar to the d-relation, Epstein $[1987,1990]$ defines the content equality relation using union set-assignment (see Section 2.3). He also demonstrates that this relation may be determined through conditions $\left(\mathrm{r}_{1}\right),\left(\mathrm{r}_{8}\right),\left(\mathrm{d}_{1}\right),\left(\mathrm{d}_{5}\right)$, and $\left(\right.$ eq $\left._{1}\right)-\left(\mathrm{eq}_{6}\right)$. 
In the following fact, some additional properties of the eq-relation are specified:

FACT 2.12. If $R$ is an eq-relation, the following conditions are satisfied:

1. $\forall_{A, B, C, D \in \mathcal{L}_{E}}((R(A, B)$ and $R(C, D)) \Rightarrow R(A \wedge C, B \wedge D))$,

2. $\forall A, B, C \in \mathcal{L}_{E}(R(A, B \wedge C) \Rightarrow R(\neg A, B \wedge \neg C))$,

3. $\forall_{A, B, C \in \mathcal{L}_{E}}(R(A, B \wedge A) \Rightarrow R(A \wedge C, B \wedge(A \wedge C)))$,

4. $\forall_{A, B, C \in \mathcal{L}_{E}}(R(A, B \wedge A) \Rightarrow R(C \wedge A, B \wedge(C \wedge A)))$,

5. $\forall_{A, B, C \in \mathcal{L}_{E}}(R(A, B \wedge A) \Rightarrow R(A \rightarrow C, B \wedge(A \rightarrow C)))$,

6. $\forall A, B, C \in \mathcal{L}_{E}(R(A, B \wedge A) \Rightarrow R(C \rightarrow A, B \wedge(C \rightarrow A)))$,

7. $\forall_{A \in \mathcal{L}_{E}} \forall_{B \in \operatorname{sub}(A)} R(A, B \wedge A)$.

Proof. For 1 see [Epstein, 1990, p. 137, Lemma 10].

For 2. Assume that $R(A, B \wedge C)$. By $\left(\mathrm{r}_{8}\right),\left(\mathrm{d}_{5}\right)$, and $\left(\mathrm{d}_{1}\right) R(\neg A, B \wedge$ $C)$. By $\left(\mathrm{r}_{1}\right) R(B, B)$, and by $\left(\mathrm{d}_{5}\right) R(C, \neg C)$. Thus, by $1, R(B \wedge C, B \wedge$ $\neg C)$. Hence, by $\left(\mathrm{d}_{1}\right), R(\neg A, B \wedge \neg C)$.

For 3. Assume that $R(A, B \wedge A)$. By $\left(\mathrm{r}_{1}\right) R(C, C)$. Thus, by 1 , $R(A \wedge C,(B \wedge A) \wedge C)$. By $\left(\mathrm{eq}_{4}\right)$ and $\left(\mathrm{r}_{8}\right) R((B \wedge A) \wedge C, B \wedge(A \wedge C))$. Hence, by $\left(\mathrm{d}_{1}\right), R(A \wedge C, B \wedge(A \wedge C))$.

For 4 we make a similar argument as for 3 .

For 5. Assume that $R(A, B \rightarrow A)$. By $\left(\mathrm{eq}_{3}\right)$ and $\left(\mathrm{d}_{1}\right) R(A, B \wedge A)$. An argument similar to that for 3 yields $R(A \wedge C, B \wedge(A \wedge C))$. Thus, by (eq $\left.)_{3}\right),\left(\mathrm{r}_{8}\right)$, and $\left(\mathrm{d}_{1}\right), R(A \rightarrow C, B \wedge(A \wedge C))$. By $\left(\mathrm{r}_{1}\right)$, (eq $\left.\mathrm{eq}_{3}\right)$, and 1 , $R(B \wedge(A \wedge C), B \wedge(A \rightarrow C))$. Hence, by $\left(\mathrm{d}_{1}\right), R(A \rightarrow C, B \wedge(A \rightarrow C))$.

For 6 we make a similar argument as for 5 .

For 7 . The argument is by induction on the complexity of formulae.

Initial step. Let $A \in \mathcal{L}_{\mathrm{E}}$ and $\mathrm{c}(A)=0$. By (eq6) $R(A, A \wedge A)$.

Inductive hypothesis. Let $n \in \mathbb{N}$. Assume that for any $A \in \mathcal{L}_{\mathrm{E}}$, if $\mathrm{c}(A) \leqslant n$, then for any $B \in \operatorname{sub}(A), R(A, B \wedge A)$.

Inductive step. Let $A \in \mathcal{L}_{\mathrm{E}}$ and $\mathrm{c}(A)=n+1$. Consider the construction of formulae in $\mathcal{L}_{\mathrm{E}}$.

Let $A=\neg C$. By $\left(\mathrm{eq}_{6}\right) R(\neg C, \neg C \wedge \neg C)$. Let $B \in \operatorname{sub}(C)$. By the inductive hypothesis $R(C, B \wedge C)$. Thus, by $2, R(\neg C, B \wedge \neg C)$.

Let $A=C * D$, where $* \in\{\wedge, \rightarrow\}$. By $\left(\mathrm{eq}_{6}\right) R(C * D,(C * D) \wedge(C *$ $D)$ ). Let $C^{\prime} \in \operatorname{sub}(C)$ and $D^{\prime} \in \operatorname{sub}(D)$. By the inductive hypothesis $R\left(C, C^{\prime} \wedge C\right)$ and $R\left(D, D^{\prime} \wedge D\right)$. Thus, by 3-6, $R\left(C * D, C^{\prime} \wedge(C * D)\right)$ and $R\left(C * D, D^{\prime} \wedge(C * D)\right)$. 
From here we should return to the problem of representation by means of eq-relations of content relationship. Facts 2.9, 2.11, and 2.12.7 provide the following conclusion:

FACT 2.13 (cf. Epstein, 1990, pp. 138-139). If $R$ is an eq-relation, for any $A, B \in \mathcal{L}_{E}, R(A, B)$ iff $\overline{\mathrm{t}}_{\mathrm{R}_{R}}(A)=\overline{\mathrm{t}}_{\mathrm{R}_{R}}(B)$.

Proof. " $\Rightarrow$ " Assume that $R(A, B)$. Since the eq-relation is symmetric, then using $\left(\mathrm{r}_{8}\right)$ we can obtain $R(B, A)$. Therefore, by the definition of $\mathrm{R}_{R}, \mathrm{R}_{R}(A, B)$ and $\mathrm{R}_{R}(B, A)$. Given Fact $2.11, \mathrm{R}_{R}$ is a d-relation. Hence, by Fact $2.9, \overline{\mathrm{t}}_{\mathrm{R}_{R}}(A)=\overline{\mathrm{t}}_{\mathrm{R}_{R}}(B)$.

" $\Leftarrow$ " Assuming that $\overline{\mathrm{t}}_{\mathrm{R}_{R}}(A)=\overline{\mathrm{t}}_{\mathrm{R}_{R}}(B)$, by Fact $2.9, \mathrm{R}_{R}(A, B)$ and $\mathrm{R}_{R}(B, A)$. Hence $R(A, C)$, where $B \in \operatorname{sub}(C)$ for some $C \in \mathcal{L}_{\mathrm{E}}$ and $R(B, D)$, where $A \in \operatorname{sub}(D)$ for some $D \in \mathcal{L}_{\mathrm{E}}$. By $\left(\mathrm{d}_{1}\right)$ and Fact 2.12.7, $R(A, B \wedge C)$ and $R(B, A \wedge D)$. Hence, by (eq $), R(A, B)$.

\subsection{Union set-assignment}

In his considerations on content relationship and in the content relationship logics that follow Epstein [1979, 1990] accepts the principle of content compositionality (with the exception of the logic DPC). As per this principle, the content of a compound sentence may be determined by the content of its components.

Epstein [1990, pp. 99-100] does not accept the principle of content compositionality uncritically; in his opinion it is not certain whether this principle should be true globally, i.e., in the case of an arbitrary understanding of content and content relationship. However, he states that in cases when it is not satisfied:

there is (or should be) some underlying, more primitive notion to which contents can be reduced and which satisfies a functional relation between the part and the whole.

[Epstein, 1990, p. 100]

In order to formally account for the principle of content compositionality Epstein introduces the notion of a union set-assignment, i.e., a set-assignment $s: \mathcal{L}_{\mathrm{E}} \longrightarrow \mathcal{P}(S)$ which satisfies the following condition for any $A \in \mathcal{L}_{\mathrm{E}}$ :

$$
s(A)=\bigcup\{s(a) \in \mathcal{P}(S): a \in \operatorname{var}(A)\} .
$$

Following Epstein [1990, pp. 65-68], it is important to highlight that the union set-assignment satisfies the following equations: 


$$
\begin{aligned}
s(\neg A) & =s(A), \\
s(A * B) & =s(A) \cup s(B),
\end{aligned}
$$

where $A, B \in \mathcal{L}_{\mathrm{E}}$ and $* \in\{\wedge, \rightarrow\}$. Thus, the union set-assignment does not allow for a representation of such an approach to content according to which the content of a compound sentence is influenced not only by the arity of the connective upon which the sentence is built but also by which specific connective is used to form the sentence.

In order to analyse the content relationship understood as the intersection (overlap) of sentence content (A), Epstein proposes the use of a non-empty union set-assignment, i.e., a union set-assignment $s$ : $\mathcal{L}_{\mathrm{E}} \longrightarrow \mathcal{P}(S)$ such that for any $A \in \operatorname{Var}, s(A) \neq \emptyset$.

Let $s: \mathcal{L}_{\mathrm{E}} \longrightarrow \mathcal{P}(S)$ be a non-empty union set-assignment. The relation $\mathrm{R}_{s} \subseteq \mathcal{L}_{\mathrm{E}} \times \mathcal{L}_{\mathrm{E}}$ is defined as follows:

$$
\mathrm{R}_{s}(A, B) \text { iff } s(A) \cap s(B) \neq \emptyset .
$$

Using this approach the following fact holds:

FACT 2.14 (Epstein, 1990, p. 69, Lemma 3). If $s: \mathcal{L}_{E} \longrightarrow \mathcal{P}(S)$ is a non-empty union set-assignment, then $\mathrm{R}_{s}$ satisfies conditions $\left(\mathrm{r}_{1}\right)-\left(\mathrm{r}_{4}\right)$ and $\left(\mathrm{r}_{8}\right)$, i.e., $\mathrm{R}_{s}$ is an sr-relation.

At this point it is important to distinguish some non-empty union setassignments. By a K-pair of non-empty union set-assignments (for short: a $K$-pair $)$ we mean an ordered pair $\left\langle s_{1}, s_{2}\right\rangle$ such that $s_{1}, s_{2}: \mathcal{L}_{\mathrm{E}} \longrightarrow$ $\mathcal{P}\left(\mathcal{L}_{\mathrm{E}} \times \mathcal{L}_{\mathrm{E}}\right)$ are non-empty union set-assignments and for any $a \in \operatorname{Var}$ the following conditions are satisfied:

$$
\begin{aligned}
& s_{1}(a) \subseteq\{a\} \times \mathcal{L}_{\mathrm{E}}, \\
& s_{2}(a) \subseteq \mathcal{L}_{\mathrm{E}} \times\{a\}, \\
& \langle a, a\rangle \in s_{1}(a) \cap s_{2}(a) .
\end{aligned}
$$

The above definition of a K-pair is based on the analysis of Krajewski [1991, pp. 19-20].

Let $\left\langle s_{1}, s_{2}\right\rangle$ be a K-pair. The relation $\mathrm{R}_{\left\langle s_{1}, s_{2}\right\rangle} \subseteq \mathcal{L}_{\mathrm{E}} \times \mathcal{L}_{\mathrm{E}}$ is defined as follows:

$$
\mathrm{R}_{\left\langle s_{1}, s_{2}\right\rangle}(A, B) \text { iff } s_{1}(A) \cap s_{2}(B) \neq \emptyset .
$$

The resulting relation is an r-relation which does not have to be symmetric:

FACT 2.15. If $\left\langle s_{1}, s_{2}\right\rangle$ is a $K$-pair, then $\mathrm{R}_{\left\langle s_{1}, s_{2}\right\rangle}$ satisfies conditions $\left(\mathrm{r}_{1}\right)$ $\left(\mathrm{r}_{7}\right)$, i.e., $\mathrm{R}_{\left\langle s_{1}, s_{2}\right\rangle}$ is an r-relation. 
Proof. For $\left(\mathrm{r}_{1}\right)$. By the definition of a K-pair, $s_{1}(A) \cap s_{2}(A) \neq \emptyset$. Hence, by the definition of $R_{\left\langle s_{1}, s_{2}\right\rangle}, R_{\left\langle s_{1}, s_{2}\right\rangle}(A, A)$.

For $\left(\mathrm{r}_{2}\right)$. We have: $R_{\left\langle s_{1}, s_{2}\right\rangle}(A, B)$, by the definition of $R_{\left\langle s_{1}, s_{2}\right\rangle}$, iff $s_{1}(A) \cap s_{2}(B) \neq \emptyset$, by the definition of union set-assignment, iff $s_{1}(\neg A) \cap$ $s_{2}(B) \neq \emptyset$, by the definition of $R_{\left\langle s_{1}, s_{2}\right\rangle}$, iff $R_{\left\langle s_{1}, s_{2}\right\rangle}(\neg A, B)$.

For $\left(\mathrm{r}_{3}\right)$. We have: $R_{\left\langle s_{1}, s_{2}\right\rangle}(A, B \wedge C)$, by the definition of $R_{\left\langle s_{1}, s_{2}\right\rangle}$, iff $s_{1}(A) \cap s_{2}(B \wedge C) \neq \emptyset$, by the definition of union set-assignment, iff $s_{1}(A) \cap\left(s_{2}(B) \cup s_{2}(C)\right) \neq \emptyset$ iff $s_{1}(A) \cap s_{2}(B \rightarrow C) \neq \emptyset$, by the definition of $R_{\left\langle s_{1}, s_{2}\right\rangle}$, iff $R_{\left\langle s_{1}, s_{2}\right\rangle}(A, B \rightarrow C)$.

For $\left(\mathrm{r}_{4}\right)$. We have: $R_{\left\langle s_{1}, s_{2}\right\rangle}(A, B \wedge C)$, by the definition of $R_{\left\langle s_{1}, s_{2}\right\rangle}$, iff $s_{1}(A) \cap s_{2}(B \wedge C) \neq \emptyset$, by the definition of union set-assignment, iff $s_{1}(A) \cap\left(s_{2}(B) \cup s_{2}(C)\right) \neq \emptyset$, by the distributivity of intersection over sum, iff $\left(s_{1}(A) \cap s_{2}(B)\right) \cup\left(s_{1}(A) \cap s_{2}(C)\right) \neq \emptyset$, by the definition of union, iff $\left(\left(s_{1}(A) \cap s_{2}(B)\right) \neq \emptyset\right.$ or $\left.\left(s_{1}(A) \cap s_{2}(C)\right) \neq \emptyset\right)$, by the definition of $R_{\left\langle s_{1}, s_{2}\right\rangle}$, iff $\left(R_{\left\langle s_{1}, s_{2}\right\rangle}(A, B)\right.$ or $\left.R_{\left\langle s_{1}, s_{2}\right\rangle}(A, C)\right)$.

For $\left(\mathrm{r}_{5}\right),\left(\mathrm{r}_{6}\right)$, and $\left(\mathrm{r}_{7}\right)$, respectively, we make a similar argument as for $\left(\mathrm{r}_{2}\right),\left(\mathrm{r}_{3}\right)$, and $\left(\mathrm{r}_{4}\right)$.

Below we present the original approach to the content dependence relation, its dual version, and the content equality relation introduced by Epstein [1987, 1990].

Let $s: \mathcal{L}_{\mathrm{E}} \longrightarrow \mathcal{P}(S)$ be a union set-assignment and $\star \in\{\supseteq, \subseteq,=\}$. The relation $\mathrm{R}_{s}^{\star} \subseteq \mathcal{L}_{\mathrm{E}} \times \mathcal{L}_{\mathrm{E}}$ is defined as follows:

$$
\mathrm{R}_{s}^{\star}(A, B) \text { iff } s(A) \star s(B) .
$$

Consequently, we arrive at the following fact:

FACT 2.16 (Epstein, 1990, p. 122, Lemma 1, p. 137, Theorem 9). Let $s: \mathcal{L}_{E} \longrightarrow \mathcal{P}(S)$ be a union set-assignment. Then:

1. $\mathrm{R}_{\bar{s}}^{\supseteq}$ satisfies conditions $\left(\mathrm{r}_{1}\right)$ and $\left(\mathrm{d}_{1}\right)-\left(\mathrm{d}_{5}\right)$, i.e., $\mathrm{R}_{\bar{s}}^{\supseteq}$ is a d-relation;

2. $\mathrm{R}_{\bar{s}} \subseteq$ satisfies conditions $\left(\mathrm{r}_{1}\right),\left(\mathrm{dd}_{1}\right),\left(\mathrm{dd}_{2}\right),\left(\mathrm{d}_{4}\right)$, and $\left(\mathrm{d}_{5}\right)$, i.e., $\mathrm{R} \frac{\subseteq}{\mathrm{s}}$ is a dd-relation;

3. $\mathrm{R}_{s}^{=}$satisfies conditions $\left(\mathrm{r}_{1}\right),\left(\mathrm{r}_{8}\right),\left(\mathrm{d}_{1}\right),\left(\mathrm{d}_{5}\right)$, and $\left(\mathrm{eq}_{1}\right)-\left(\mathrm{eq} \mathrm{q}_{6}\right)$, i.e., $\mathrm{R}_{s}^{=}$ is an eq-relation.

\subsection{Comparison of two approaches to the representation of content}

In this section, we will demonstrate that the transition between the relational approach and the functional approach does not lead to any loss 
of information regarding the content relationship of sentences. Firstly, it is important to specify a fact relating to the content relationship analysed using sr-relations:

FACT 2.17 (Epstein, 1990, p. 69, Lemma 4). 1. Let $R$ be an sr-relation and $Q \subseteq \mathcal{L}_{E} \times \mathcal{L}_{E}$ be a relation defined as follows:

$$
\mathrm{Q}(A, B) \text { iff } \mathrm{s}_{R}(A) \cap \mathrm{s}_{R}(B) \neq \emptyset .
$$

Then $Q=R$.

2. Let $s: \mathcal{L}_{E} \longrightarrow \mathcal{P}(S)$ be a non-empty union set-assignment and $t: \mathcal{L}_{E}$ $\longrightarrow \mathcal{P}\left(\mathcal{L}_{E}+\mathcal{L}_{E}\right)$ be a function defined as follows:

$$
t(A):=\left\{\{A, B\} \in \mathcal{L}_{E}+\mathcal{L}_{E}: \mathrm{R}_{s}(A, B)\right\},
$$

i.e., $t=\mathrm{s}_{\mathrm{R}_{s}}$. Then, for any $A, B \in \mathcal{L}_{E}$ :

$$
t(A) \cap t(B) \neq \emptyset \text { iff } s(A) \cap s(B) \neq \emptyset .
$$

A counterpart of Fact 2.17 also holds for an r-relation than does not need to be symmetric:

FACT 2.18. 1. Let $R$ be an r-relation and $Q \subseteq \mathcal{L}_{E} \times \mathcal{L}_{E}$ be a relation defined as follows:

$$
Q(A, B) \text { iff } \mathrm{s}_{1_{R}}(A) \cap \mathrm{s}_{2_{R}}(B) \neq \emptyset .
$$

Then $Q=R$.

2. Let $\left\langle s_{1}, s_{2}\right\rangle$ be a $K$-pair and $t_{1}, t_{2}: \mathcal{L}_{E} \longrightarrow \mathcal{P}\left(\mathcal{L}_{E} \times \mathcal{L}_{E}\right)$ be functions defined as follows:

$$
\begin{aligned}
& t_{1}(A):=\left\{\langle A, B\rangle \in \mathcal{L}_{E} \times \mathcal{L}_{E}: \mathrm{R}_{\left\langle s_{1}, s_{2}\right\rangle}(A, B)\right\} \\
& t_{2}(A):=\left\{\langle B, A\rangle \in \mathcal{L}_{E} \times \mathcal{L}_{E}: \mathrm{R}_{\left\langle s_{1}, s_{2}\right\rangle}(B, A)\right\},
\end{aligned}
$$

i.e., $\left\langle t_{1}, t_{2}\right\rangle=\left\langle\mathrm{s}_{\mathrm{R}_{\left\langle s_{1}, s_{2}\right\rangle}}, \mathrm{s} 2_{\mathrm{R}_{\left\langle s_{1}, s_{2}\right\rangle}}\right\rangle$. Then, for any $A, B \in \mathcal{L}_{E}$ :

$$
t_{1}(A) \cap t_{2}(B) \neq \emptyset \text { iff } s_{1}(A) \cap s_{2}(B) \neq \emptyset .
$$

Proof. For 1. We have: $Q(A, B)$, by the definition of $Q, \mathrm{~s}_{1_{R}}(A) \cap$ $\mathrm{s}_{2_{R}}(B) \neq \emptyset$, by Fact 2.5 , iff $R(A, B)$.

For 2. By Fact $2.15 \mathrm{R}_{\left\langle s_{1}, s_{2}\right\rangle}$ is an r-relation. We have: $t_{1}(A) \cap t_{2}(B) \neq$ $\emptyset$, by the definition of $t_{1}, t_{2}$ and by Fact 2.5 , iff $\mathrm{R}_{\left\langle s_{1}, s_{2}\right\rangle}(A, B)$, by the definition of $\mathrm{R}_{\left\langle s_{1}, s_{2}\right\rangle}$, iff $s_{1}(A) \cap s_{2}(B) \neq \emptyset$. 
It should be emphasised that the above comparison is not a real comparison between the relational approach and the functional approach. Indeed, with regards to the latter, in the previous section we only accounted for union set-assignments. The $\mathrm{s}_{R}$ functions where $R$ is an sr-relation and $\mathrm{s}_{R}, \mathrm{~s} 2 R$ where $R$ is an r-relation are not that kind of functions. However, this is not a problem because the definitions given by Epstein can be modified easily. In the following discussion we will define functions determined for appropriate relations, which will be union set-assignments.

Let $R$ be an sr-relation. The function $\mathrm{s}_{R}\left\lceil\mathrm{Var}_{\mathrm{v}}\right.$ is extended to a function $\mathrm{s}_{R}^{\prime}$ onto the entire set $\mathcal{L}_{\mathrm{E}}$. For any $A \in \mathcal{L}_{\mathrm{E}}$, we define:

$$
\mathrm{s}_{R}^{\prime}(A):=\bigcup\left\{\mathrm{s}_{R}(a) \in \mathcal{P}\left(\mathcal{L}_{\mathrm{E}}+\mathcal{L}_{\mathrm{E}}\right): a \in \operatorname{var}(A)\right\} .
$$

Function $s_{R}^{\prime}$ is a non-empty union set-assignment. Moreover, the following fact holds:

FACT 2.19. Let $R$ be an sr-relation. Then, for any $A, B \in \mathcal{L}_{E}, \mathrm{~s}_{R}^{\prime}(A) \cap$ $\mathrm{s}_{R}^{\prime}(B) \neq \emptyset$ iff $\mathrm{s}_{R}(A) \cap \mathrm{s}_{R}(B) \neq \emptyset$.

Proof. " $\Rightarrow$ " Assume that $\mathrm{s}_{R}^{\prime}(A) \cap \mathrm{s}_{R}^{\prime}(B) \neq \emptyset$. If $A=B$, then using $\left(\mathrm{r}_{1}\right), R(A, B)$. Hence, by Fact $2.4, \mathrm{~s}_{R}(A) \cap \mathrm{s}_{R}(B) \neq \emptyset$. Suppose that $A \neq B$. By the initial assumption, there exist $C, D \in \mathcal{L}_{\mathrm{E}}$ such that $\{C, D\} \in \mathrm{s}_{R}^{\prime}(A) \cap \mathrm{s}_{R}^{\prime}(B)$. Given the definition of $\mathrm{s}_{R}^{\prime}$, let $a \in \operatorname{var}(A)$ and $b \in \operatorname{var}(B)$ be such that that $\{C, D\} \in \mathrm{s}_{R}^{\prime}(a)=\mathrm{s}_{R}(a)$ and $\{C, D\} \in$ $\mathrm{s}_{R}^{\prime}(b)=\mathrm{s}_{R}(b)$. Therefore, $C=a$ and $D=b$, or $C=b$ and $D=a$, or $C=a=b$, or $D=a=b$. Thus, using the definitions of $\mathrm{s}_{R}$ and the sr-relation, either $R(a, b)$ or $R(b, a)$. If $R(b, a)$, then $R(a, b)$ because of $\left(\mathrm{r}_{8}\right)$. Hence, $R(a, b)$. Therefore, $\exists_{a \in \operatorname{var}(A)} \exists_{b \in \operatorname{var}(B)} R(a, b)$ and, given 2.1, $R(A, B)$. Thus, by Fact $2.4, \mathrm{~s}_{R}(A) \cap \mathrm{s}_{R}(B) \neq \emptyset$.

" $\Leftarrow$ " Assume that $\mathrm{s}_{R}(A) \cap \mathrm{s}_{R}(B) \neq \emptyset$. Hence, by Fact 2.4, $R(A, B)$. Therefore, by Fact 2.1, $\exists_{a \in \operatorname{var}(A)} \exists_{b \in \operatorname{var}(B)} R(a, b)$. Let $a \in \operatorname{var}(A), b \in$ $\operatorname{var}(B)$, and $R(a, b)$. By $\left(\mathrm{r}_{8}\right), R(b, a)$. Hence, by the definition of $\mathrm{s}_{R}$ and $\mathrm{s}_{R}^{\prime},\{a, b\} \in \mathrm{s}_{R}(a)=\mathrm{s}_{R}^{\prime}(a)$ and $\{a, b\} \in \mathrm{s}_{R}(b)=\mathrm{s}_{R}^{\prime}(b)$. Thus, using the definition of $s_{R}^{\prime}, \mathrm{s}_{R}^{\prime}(A) \cap \mathrm{s}_{R}^{\prime}(B) \neq \emptyset$.

The construction of an $r$-relation $R$ that is not necessarily symmetric is similar. The functions $s 1_{R}\left\lceil V_{\text {ar }}\right.$ and $s 2_{R}\left\lceil V_{\text {ar }}\right.$ are extended to $s 1_{R}^{\prime}$ and s2 ${ }_{R}^{\prime}$, respectively, onto the entire set $\mathcal{L}_{\mathrm{E}}$. For any $A \in \mathcal{L}_{\mathrm{E}}$, we define:

$$
\begin{aligned}
& \mathrm{s}_{R}^{\prime}(A):=\bigcup\left\{\mathrm{s} 1_{R}(a) \in \mathcal{P}\left(\mathcal{L}_{\mathrm{E}} \times \mathcal{L}_{\mathrm{E}}\right): a \in \operatorname{var}(A)\right\}, \\
& \mathrm{s}_{R}^{\prime}(A):=\bigcup\left\{\mathrm{s} 2_{R}(a) \in \mathcal{P}\left(\mathcal{L}_{\mathrm{E}} \times \mathcal{L}_{\mathrm{E}}\right): a \in \operatorname{var}(A)\right\} .
\end{aligned}
$$


The ordered pair $\left\langle\mathrm{s}_{R}^{\prime}, \mathrm{sz}_{R}^{\prime}\right\rangle$ is a K-pair. Moreover, the following fact holds:

FACT 2.20. Let $R$ be an r-relation. For any $A, B \in \mathcal{L}_{E}$, $\mathrm{s}_{R}^{\prime}(A) \cap \mathrm{sz}_{R}^{\prime}(B)$ $\neq \emptyset$ iff $\mathrm{s} 1_{R}(A) \cap \mathrm{s} 2_{R}(B) \neq \emptyset$.

Proof. " $\Rightarrow$ " Assume that $\mathrm{s}_{R}^{\prime}(A) \cap \mathrm{s} 2_{R}^{\prime}(B) \neq \emptyset$. If $A=B$, then using $\left(\mathrm{r}_{1}\right), R(A, B)$. Hence, by Fact $2.5, \mathrm{~s}_{R}(A) \cap \mathrm{s} 2_{R}(B) \neq \emptyset$. Suppose that $A \neq B$. Given the initial assumption, there are $C, D \in \mathcal{L}_{\mathrm{E}}$ such that $\langle C, D\rangle \in \mathrm{s}_{R}^{\prime}(A) \cap \mathrm{s}_{R}^{\prime}(B)$. By the definition of $\mathrm{s}_{R}^{\prime}, \mathrm{s}_{R}^{\prime}$ let $a \in \operatorname{var}(A)$ and $b \in \operatorname{var}(B)$ be such that $\langle C, D\rangle \in \mathrm{s}_{R}^{\prime}(a)=\mathrm{s} 1_{R}(a)$ and $\langle C, D\rangle \in$ $\mathrm{s}_{R}^{\prime}(b)=\mathrm{s} 2_{R}(b)$. Hence, by the definition of $\mathrm{s} 1_{R}, \mathrm{~s} 2_{R}, C=a$ and $D=b$. Thus, $R(a, b)$. Therefore, $\exists_{a \in \operatorname{var}(A)} \exists_{b \in \operatorname{var}(B)} R(a, b)$. Consequently, by Fact 2.1, $R(A, B)$. Given the above, by Fact $2.5, \mathrm{~s}_{R}(A) \cap \mathrm{s}_{R}(B) \neq \emptyset$.

" $\Leftarrow$ " We now assume that $\mathrm{s}_{R}(A) \cap \mathrm{s} 2_{R}(B) \neq \emptyset$. Hence, by Fact 2.5 , $R(A, B)$. Therefore, by Fact $2.5, \exists_{a \in \operatorname{var}(A)} \exists_{b \in \operatorname{var}(B)} R(a, b)$. Let $a \in$ $\operatorname{var}(A), b \in \operatorname{var}(B)$ and $R(a, b)$. Hence, by the definition of $\mathrm{s}_{R}, \mathrm{~s} 2_{R}$ and $\mathrm{s}_{R}^{\prime}, \mathrm{s}_{R}^{\prime},\langle a, b\rangle \in \mathrm{s}_{R}(a)=\mathrm{s}_{R}^{\prime}(a)$ and $\langle a, b\rangle \in \mathrm{s} 2_{R}(b)=\mathrm{s}_{R}^{\prime}(b)$. Thus, according to the definition of $\mathrm{s}_{R}^{\prime}, \mathrm{s}_{R}^{\prime}, \mathrm{s}_{R}^{\prime}(A) \cap \mathrm{s}{ }_{R}^{\prime}(B) \neq \emptyset$.

The presented analysis of content relationship understood as inclusion of content has been stated in both the relational and functional language. Therefore, also in this case one might enquire about the preservation of information on the content relationship when passing from the relational to the functional approach or vice versa. Firstly, we will examine the content relationship understood as inclusion (B1).

FACT 2.21. 1. Let $R$ be a $d$-relation and $Q \subseteq \mathcal{L}_{E} \times \mathcal{L}_{E}$ be a relation defined as follows:

$$
Q(A, B) \text { iff } \overline{\mathrm{t}}_{R}(B) \subseteq \overline{\mathrm{t}}_{R}(A)
$$

Then $Q=R$.

2. Let $s: \mathcal{L}_{E} \longrightarrow \mathcal{P}(S)$ be a union set-assignment and $t: \mathcal{L}_{E} \longrightarrow$ $\mathcal{P}\left(\mathcal{L}_{E}\right)$ be a function defined as follows:

$$
t(A):=\mathcal{L}_{E} \backslash \mathrm{t}_{\mathrm{R}_{s}^{\supset}}(A),
$$

i.e., $t=\overline{\mathrm{t}}_{\mathrm{R} \frac{\supset}{s}}$. Then, for any $A, B \in \mathcal{L}_{E}$ :

$$
t(A) \subseteq t(B) \text { iff } s(A) \subseteq s(B)
$$


Proof. For 1. Follows from Fact 2.9.

For 2. By Fact 2.16.1 $\mathrm{R} \frac{\supset}{s}$ is a d-relation. We have: $t(A) \subseteq t(B)$, by the definition of $t$ and by Fact 2.9, iff $\mathrm{R} \frac{\supset}{s}(B, A)$, by the definition of $\mathrm{R} \frac{\supset}{s}$, iff $s(A) \subseteq s(B)$.

The case of content relationship understood as inclusion in the sense of (B2) is analogous:

FACT 2.22. 1. Let $R$ be a dd-relation and $Q \subseteq \mathcal{L}_{E} \times \mathcal{L}_{E}$ be a relation defined as follows:

$$
Q(A, B) \text { iff } \overline{\mathrm{u}}_{R}(A) \subseteq \overline{\mathrm{u}}_{R}(B) .
$$

Then $Q=R$.

2. Let $s: \mathcal{L}_{E} \longrightarrow \mathcal{P}(S)$ be a union set-assignment and $t: \mathcal{L}_{E} \longrightarrow$ $\mathcal{P}\left(\mathcal{L}_{E}\right)$ be a function defined as follows:

$$
t(A):=\mathcal{L}_{E} \backslash \mathrm{u}_{\mathrm{R}} \frac{\subseteq}{s}(A),
$$

i.e., $t=\overline{\mathrm{u}}_{\mathrm{R}_{\bar{s}}}$. Then, for any $A, B \in \mathcal{L}_{E}$ :

$$
t(A) \subseteq t(B) \text { iff } s(A) \subseteq s(B) .
$$

Proof. For 1. Follows from Fact 2.10.

For 2. Given Fact 2.16.2, $\mathrm{R} \subseteq$ is a dd-relation. Here, we have $t(A) \subseteq$ $t(B)$, by the definition of $t$ and by Fact 2.10 , iff $\mathrm{R}_{s}^{\subseteq}(A, B)$, by the definition of $\mathrm{R}_{\bar{s}}$, iff $s(A) \subseteq s(B)$.

The case of content relationship understood as equality in the sense of point (B3) is similar:

FACT 2.23. 1. Let $R$ be an eq-relation and $Q \subseteq \mathcal{L}_{E} \times \mathcal{L}_{E}$ be a relation defined as follows:

$$
\mathrm{Q}(A, B) \text { iff } \overline{\mathrm{t}}_{\mathrm{R}_{R}}(A)=\overline{\mathrm{t}}_{\mathrm{R}_{R}}(B) .
$$

Then $Q=R$.

2. Let $s: \mathcal{L}_{E} \longrightarrow \mathcal{P}(S)$ be a union set-assignment and $t: \mathcal{L}_{E} \longrightarrow$ $\mathcal{P}\left(\mathcal{L}_{E}\right)$ be a function defined as follows:

$$
t(A):=\mathcal{L}_{E} \backslash \mathrm{t}_{\mathrm{R}_{\mathrm{R}}^{\bar{s}}},
$$

i.e., $t=\overline{\mathrm{t}}_{\mathrm{R}_{\mathrm{Q}}}$. Then, for any $A, B \in \mathcal{L}_{E}$ :

$$
t(A)=t(B) \text { iff } s(A)=s(B) .
$$


Proof. For 1. Follows from Fact 2.13.

For 2. By Fact 2.16.3 $\mathrm{R}_{s}^{=}$is an eq-relation. We have: $t(A)=t(B)$, by the definition of $t$ and by Fact 2.13 , iff $\mathrm{R}_{s}^{=}(A, B)$, by the definition of $\mathrm{R}_{s}^{=}$, if $s(A)=s(B)$.

Let us notice that functions we use in the comparison of relational and functional approach to analysis of content relationship understood as inclusion of content are union set-assignments. We have the following fact:

FACT 2.24. If $R$ is a d-relation, then $\overline{\mathrm{t}}_{R}$ is a union set-assignment.

Proof. Let $A, B \in \mathcal{L}_{\mathrm{E}}$. We have: $B \in \bigcup\left\{\overline{\mathrm{t}}_{R}(a) \in \mathcal{P}\left(\mathcal{L}_{\mathrm{E}}\right): a \in\right.$ $\operatorname{var}(A)\}$ iff $\exists_{a \in \operatorname{var}(A)} B \in \overline{\mathrm{t}}_{R}(a)$, by definition of $\overline{\mathrm{t}}_{R}$, iff $\exists_{a \in \operatorname{var}(A)} B \notin \mathrm{u}_{R}(a)$, by definition of $\overline{\mathrm{t}}_{R}$, iff $\exists_{a \in \operatorname{var}(A)} \sim R(B, a)$, by law of classical logic, iff $\sim \forall_{a \in \operatorname{var}(A)} R(B, a)$, by Fact 2.7, iff $\sim R(B, A)$, by definition of $\mathrm{t}_{R}$, iff $B \notin \mathrm{t}_{R}(A)$, by definition of $\overline{\mathrm{t}}_{R}$, iff $B \in \overline{\mathrm{t}}_{R}(A)$.

The counterpart of Fact 2.24 obviously holds for dd-relation, so we can prove the following fact:

FACT 2.25. If $R$ is a $d d$-relation, then $\overline{\mathrm{u}}_{R}$ is a union set-assignment.

Proof. We make a similar argument as for Fact 2.24, we use ( $\ddagger$ ) and the counterpart of Fact 2.7.

\subsection{Epstein's Logic (Goal 1)}

Point 2 of Epstein's Programme states that the first goal (see goal 1) of the programme is to introduce propositional logics based on the analysis of content relationship. Epstein [1979] [see also Epstein, 1987, 1990; Krajewski, 1991] determines two types of such logics in the language $\mathcal{L}_{\mathrm{E}}$. These logics, which may be considered to be special cases of the relating logic (see Section 3.1), are as follows:

- relatedness logic: $\mathbf{R}$ and $\mathbf{S}$ (motivated by the concept of content relationship as non-empty intersection of contents (A)),

- dependence logic: D, DD, Eq and DPC (motivated by the concept of content relationship as inclusion of contents (B1)-(B3)).

Epstein's logics may be determined either by relational semantics or by functional semantics. The starting point is an introduction to the notion of relational semantics, founded on models based on the extension of the relatedness relation. 
By a model over an extension of relatedness relation (for short: an err-model) we mean an ordered pair $\langle v, R\rangle$ such that $v: \operatorname{Var} \longrightarrow\{1,0\}$ is a valuation of propositional variables and $R \subseteq \mathcal{L}_{\mathrm{E}} \times \mathcal{L}_{\mathrm{E}}$ is an arbitrary reflexive relation; in other words, $R$ satisfies at least condition $\left(\mathrm{r}_{1}\right)$.

An err-model $\langle v, R\rangle$ will be called:

- an $r$-model iff $R$ is an r-relation,

- an sr-model iff $R$ is an sr-relation,

- a $d$-model iff $R$ is a d-relation,

- a $d d$-model iff $R$ is a dd-relation,

- an eq-model iff $R$ is an eq-relation,

- a cl-model iff $R=\models \mathcal{L}_{\mathrm{E}}$.

For any err-model $\mathfrak{M}=\langle v, R\rangle$ formula $A \in \mathcal{L}_{\mathrm{E}}$ is true in $\mathfrak{M}$ (in symb.: $\mathfrak{M} \models A$ ) iff for any $B, C \in \mathcal{L}_{\mathrm{E}}$ :

$$
\begin{array}{ll}
v(A)=1, & \text { if } A \in \operatorname{Var}, \\
\text { not } \mathfrak{M} \models B \quad \text { in symb.: } \mathfrak{M} \forall B), & \text { if } A=\neg B, \\
\mathfrak{M} \models B \text { and } \mathfrak{M} \models C, & \text { if } A=B \wedge C, \\
\mathfrak{M} \forall=B \text { or } \mathfrak{M} \models C, \text { and } R(B, C), & \text { if } A=B \rightarrow C .
\end{array}
$$

The notions of semantic consequence and validity are defined in a standard way with respect to the true formula in an err-model, i.e., for any set $X \cup\{A\} \subseteq \mathcal{L}_{\mathrm{E}}$ and any set of err-models $\mathbf{M}$ :

- $\quad A$ is valid in $\mathbf{M}$ iff $\forall_{\mathfrak{M} \in \mathbf{M} \mathfrak{M}} \models A$.

- $A$ is a semantic consequence of $X$ in $\mathbf{M}$ iff $\forall_{\mathfrak{M} \in \mathbf{M}}\left(\forall_{B \in X} \mathfrak{M} \models B \Rightarrow\right.$ $\mathfrak{M} \models A$ ).

The next step involves defining functional models. To this end, the notion of model based on set-assignment is introduced below. In this context, a model based on a set-assignment (for short: an sa-model) refers to an ordered triple $\langle v, s, t\rangle$ where $v: \operatorname{Var} \longrightarrow\{1,0\}$ is a valuation of propositional variables, and $s: \mathcal{L}_{\mathrm{E}} \longrightarrow \mathcal{P}(S)$ and $t: \mathcal{L}_{\mathrm{E}} \longrightarrow \mathcal{P}(T)$ are set-assignments.

An sa-model $\langle v, s, t\rangle$ will be called:

- a $u$-model iff $s=t$ and $s$ is a union set-assignment,

- a nu-model iff $s=t$ and $s$ is a non-empty union set-assignment,

- a $K$-model iff $\langle s, t\rangle$ is a K-pair,

- a cn-model iff $s=t$ and $s=\mathrm{Cn}_{\mathcal{L}_{\mathrm{E}}}$.

Unlike in the case of relational semantics, we introduce different definitions of the notion of true formula in a model by providing different 
conditions for an implication to be true. For any sa-model $\mathfrak{M}=\langle v, s, t\rangle$, formula $A \in \mathcal{L}_{\mathrm{E}}$ is true in $\mathfrak{M}$ iff for any $B, C \in \mathcal{L}_{\mathrm{E}}$ :

- If $A$ is a propositional variable or is constructed using negation $\neg$ or conjunction $\wedge$, we accept standard conditions:

$$
\begin{array}{ll}
v(A)=1, & \text { if } A \in \mathrm{Var}, \\
\text { not } \mathfrak{M} \models B \quad(\text { in symb.: } \mathfrak{M} \not \models B), & \text { if } A=\neg B, \\
\mathfrak{M} \models B \text { and } \mathfrak{M} \models C, & \text { if } A=B \wedge C .
\end{array}
$$

- If $A$ is constructed using implication $\rightarrow$, we accept one of the following conditions:

$$
\begin{array}{lll}
\mathfrak{M} \not=B \text { or } \mathfrak{M} \models C, \text { and } s(B) \cap t(C) \neq \emptyset, & \text { if } A:=B \rightarrow C, & (\cap) \\
\mathfrak{M} \not \models B \text { or } \mathfrak{M} \models C, \text { and } s(B) \supseteq t(C), & \text { if } A:=B \rightarrow C, & (\supseteq) \\
\mathfrak{M} \not \models B \text { or } \mathfrak{M} \models C, \text { and } s(B) \subseteq t(C), & \text { if } A:=B \rightarrow C, & (\subseteq) \\
\mathfrak{M} \not=B \text { or } \mathfrak{M} \models C, \text { and } s(B)=t(C), & \text { if } A:=B \rightarrow C . & (=)
\end{array}
$$

Semantic consequence and validity for a given set of sa-models are defined in a standard way, i.e., as above by assuming one or another definition of truth in a model.

Let us now define Epstein's logics:

- The logic $\mathbf{R}$ is defined by the set of all r-models. Moreover, we can define it by the set of all K-models, accepting the definition of true formulae in the model with condition $(\cap)$.

- The logic $\mathbf{S}$ is defined by the set of all sr-models. Moreover, we can define it by the set of all nu-models, accepting the definition of true formulae in the model with condition $(\cap)$.

- The logic $\mathbf{D}$ is defined by the set of all d-models. Moreover, we can define it by the set of all $\mathrm{u}$-models, accepting the definition of true formulae in the model with condition $(\supseteq)$.

- The logic DD is defined by the set of all dd-models. Moreover, we can define it by the set of all u-models, accepting the definition of true formulae in the model with condition $(\subseteq)$.

- The logic Eq is defined by the set of all eq-models. Moreover, we can define it by the set of all u-models, accepting the definition of true formulae in the model with condition $(=)$.

- The logic DPC is defined by the set of all cl-models. Moreover, we can define it by the set of all cn-models, accepting the definition of true formulae in the model with condition $(\supseteq){ }^{8}$

${ }^{8}$ Epstein [1990] also considers logics $\mathbf{D P C}$, for all $n \geqslant 1$. These logics are 
It should be emphasised that Epstein [1990] defines his relatedness logic $\mathbf{R}$ only by means of relational models. Functional models for the logic $\mathbf{R}$ may be defined based on Krajewski's [1991] analysis. The dependence logics, in turn, are defined mainly by functional models, although Epstein $[1987,1990]$ notes that these logics can be defined by appropriate relational models and specifies what relational conditions are needed for that.

Below we will present selected results on relatedness logics and dependence logics. Apart from those obtained by Epstein, which we have already discussed, and those which will be described in the next section, it is important to mention first and foremost the appropriate axiomatisation of the logics $\mathbf{R}$ and $\mathbf{S}$ presented in [Epstein, 1979] as well as that of the logics D, DD and Eq presented in [Epstein, 1990]. Another important result is the functional completeness of the set $\{\neg, \rightarrow\}$, which is proven for the logics $\mathbf{S}$ and $\mathbf{D}$ [Epstein, 1990]. Moreover, for the dependence $\operatorname{logics} \mathbf{D}, \mathbf{D D}$ and $\mathbf{E q}$, an algebraic interpretation has been found in [Epstein, 1987]. In [Epstein, 1990] it has been shown that no many-valued interpretation with a finite set of values exists for the following logics: $\mathbf{R}, \mathbf{S}, \mathbf{D}, \mathbf{D D}$ and Eq.

It is also worth mentioning Epstein's work [Epstein, 2005] in which he describes the so-called paraconsistent counterparts of logics S, D, DD, and Eq, i.e., the logics PS, PD, PDD, and PEq. These logics are obtained by modifying the notion of semantic consequence in the case where there is a non-empty set of premises. Let $X \cup\{A\} \subseteq \mathcal{L}_{\mathrm{E}}$ and $X \neq \emptyset$. The following definitions apply:

- $X \models_{\mathbf{P S}} A$ iff there exist $B_{1}, \ldots B_{n} \in X$ such that for an arbitrary nu-model $\langle v, s\rangle$ of the logic $\mathbf{S}$ :

- $\langle v, s\rangle \models X \Rightarrow\langle v, s\rangle \models A$,

- $s\left(B_{1} \wedge \ldots \wedge B_{n}\right) \cap s(A) \neq \emptyset$.

- $X \models_{\mathbf{P D}} A$ iff there exist $B_{1}, \ldots B_{n} \in X$ such that for an arbitrary u-model $\langle v, s\rangle$ of the logic $\mathbf{D}$ :

- $\langle v, s\rangle \models X \Rightarrow\langle v, s\rangle \models A$,

- $s\left(B_{1} \wedge \ldots \wedge B_{n}\right) \supseteq s(A)$.

defined inductively. Firstly, $\mathbf{D P C} \mathbf{C}_{1}:=$ DPC. Next, assume that $\mathbf{D P C} \mathbf{C}_{n}$ is defined; the semantic consequence relation (or respectively, the semantic consequence operation) defined with respect to the class of models $\mathbf{D P C}$ is denoted by $\models{ }_{\mathbf{D P C}}\left(\mathbf{C n}_{\mathbf{D P C}_{n}}\right.$, respectively). Therefore, $\mathbf{D P C} \mathbf{C}_{n+1}$ is defined in the same manner as DPC but with models based on the relation $\models{ }_{\mathbf{D P C}}$ or the function $\mathbf{C n}_{\mathbf{D P C}}$. 
- $X \models_{\text {PDD }} A$ iff there exist $B_{1}, \ldots B_{n} \in X$ such that for an arbitrary u-model $\langle v, s\rangle$ of the logic DD:

- $\langle v, s\rangle \models X \Rightarrow\langle v, s\rangle \models A$,

- $s\left(B_{1} \wedge \ldots \wedge B_{n}\right) \subseteq s(A)$.

- $X \models_{\text {PEq }} A$ iff there exist $B_{1}, \ldots B_{n} \in X$ such that for an arbitrary u-model $\langle v, s\rangle$ of the logic Eq:

- $\langle v, s\rangle \models X \Rightarrow\langle v, s\rangle \models A$,

- $s\left(B_{1} \wedge \ldots \wedge B_{n}\right)=s(A)$.

It is important to note that Epstein's proposals correspond with the deliberations on the so-called containment logics discussed in detail in [Ferguson, 2017].

Epstein and Krajewski [2004] have also presented selected properties of first order relatedness logic. An extension of relatedness logic to a first order language was described for the first time in [Krajewski, 1986] and later in [Krajewski, 1991]. The latter work defines set-assignments with respect to r-relation, which provides a basis for defining sa-models for the logic R. Epstein's Programme is also formulated.

Proposed applications and philosophical foundations of relatedness logic have been presented by Walton [1979a]. Obviously, the problem of interpreting conditional sentences so as to account for the content relationship between sentences, is stated as the key motive for the creation of Epstein's logics. The problem of content relationship between sentences, as well as attempts to express such relationships formally in a manner similar to that of Epstein, has been discussed by Walton [1982] in terms of argumentation theory and analysis of reasoning.

Furthermore, a critique of the possibilities of interpreting relatedness logic as a theory of inference and understanding of the relatedness logic implication as a certain description of the entailment was presented by Iseminger [1986].

Walton [1979a] pointed out that the relatedness relation could also be employed in epistemic logic. In his opinion, in order to eliminate the fundamental problem of epistemic logic, i.e., the so-called problem of logical omniscience, one can replace the material implication with the implication of relatedness logic. The source of the problem would have been the impossibility of differentiating in epistemic logic between sentences that are content related and those that have nothing in common. According to Walton, the relatedness of sentences plays an important role in epistemic contexts because an agent is not aware of all the consequences 
of its knowledge, being only aware of certain immediate consequences which it can relate, in terms of content, to its own knowledge [cf. Fagin and Halpern, 1988]. Moreover, in [Walton, 1979a] the author hinted at the possibility of using the relatedness relation in formal action theory for the analysis of causation and sequences of actions. This subject is examined in further detail in [Walton, 1979b, 1985].

In [Walton, 1979a], the possibility of using the relatedness relation to describe the analytic implication of William Parry according to Michael Dunn's approach was suggested for the first time. The work [Ledda, Paoli and Baldi, 2019] is also related to this observation, presenting various algebraic interpretations of a demodalized analytic implication understood as an implication of the logic D. In an earlier period Paoli alone presented a series of results related to Epstein's logics. In [Paoli, 1996], for example, he provided constructive proof of completeness for the logic S (for an alternative proof see Klonowski, 2018) ${ }^{9}$ and determined the limited principle of substitution of equivalent formulae for S. An analysis of the FDE-fragment of $\mathbf{S}$ in an algebraic, matrix, and axiomatic setting is presented in [Paoli, 1993]. These results were extended to the FDE-fragments of $\mathbf{R}, \mathbf{D}, \mathbf{D D}$ and Eq in [Paoli, 2007], which also includes tableau systems for these FDE-fragments.

In the 1980s and 1990s, Epstein's logics and other related logics were described in various syntactic (proof-theoretic) ways. Aside from the axiomatic systems introduced by Epstein, Carnielli [1987] defined tableau systems for S, R, D, and DD. Meanwhile, Fariñas del Cerro and Lugardon [1991] defined sequent systems for a certain modification of the dependence logics $\mathbf{D}$ and $\mathbf{D D}$, and $\mathbf{E q}$, determined by u-models defined over a selected union set-assignment. In [Fariñas del Cerro and Lugardon, 1997] logics of this kind were extended onto a first order language and sequent systems for these extensions were defined.

\subsection{Set-assignment semantics for non-classical logics (Goal 2)}

Point 2 of Epstein's Programme states the second goal (see goal 2) of the programme: to define a new type of semantics for various non-classical logics motivated by an analysis of content relationship. An example

9 The Torun Logic Group became familiar with Paoli's works from [Paoli, 1993, 1996, 2007] as well as the works of his team from the University in Cagliari after Jarmużek's research stay in Cagliari in 2019. 
of such semantics is provided by a functional semantics based on setassignments, i.e., the set-assignment semantics, examples of which are described in the previous section using the notion of a sa-model. Epstein [1990] defines set-assignment semantics for the following non-classical logics:

- modal logic: S4, S5, S4Grz, T, KTB (called the logic B by Epstein), K, QT, G, G* (ch. VI, pp. 145-194);

- intuitionistic logic: IPL, Johansson's MIL (ch. VII, pp. 195-228);

- multi-valued logic: $\mathbf{L}_{\mathbf{3}}, \mathbf{L}_{n}, \mathbf{\mathbf { L }}_{\aleph_{0}}, \mathbf{K}_{\mathbf{3}}, \mathbf{G}_{\mathbf{3}}, \mathbf{G}_{\aleph_{0}}$ (ch. VIII, pp. 229-262);

- paraconsistent logic: $\mathbf{J}_{\mathbf{3}}$ (ch. IX, pp. 263-287).

As an example, we will now describe Epstein's functional semantics for the logics $\mathbf{S} \mathbf{4}$ and $\mathbf{T}$. We will also make some comments on the models for the logic $\mathbf{K}$.

Consider the following modal language:

$$
\mathcal{L}_{\mathrm{M}}:=\langle\operatorname{Var}, \neg, \wedge, \square,(,)\rangle .
$$

The set of formulae in $\mathcal{L}_{\mathrm{M}}$ is defined in a standard way and is denoted by $\mathcal{L}_{\mathrm{M}}$. We will use the following formula abbreviations:

$$
\begin{gathered}
A \supset B:=\neg(A \wedge \neg B), \\
\vee A:=\neg \square \neg A .
\end{gathered}
$$

It should be emphasised that Epstein [1990, pp. 145-149] also defines modal logics using $\mathcal{L}_{\mathrm{E}}$. In this case, implication $\rightarrow$ is meant to allow for defining modality $\square$ :

$$
\square A:=\neg A \rightarrow A .
$$

In order to define models for selected modal logics, Epstein introduces the following conditions for the set-assignment $s: \mathcal{L}_{\mathrm{M}} \longrightarrow \mathcal{P}(S)$, for any $A, B \in \mathcal{L}_{\mathrm{M}}$ :

$$
\begin{aligned}
s(A \wedge B) & =s(A) \cap s(B), \\
s(\neg A) & =S \backslash s(A) .
\end{aligned}
$$

Any set-assignment which satisfies such conditions will be called a Boolean set-assignment.

An arbitrary sa-model $\langle v, o, s\rangle$ will be called an m-model iff:

- $o: \mathcal{L}_{\mathrm{M}} \longrightarrow \mathcal{P}(O)$ is a Boolean set-assignment,

- $s: \mathcal{L}_{\mathrm{M}} \longrightarrow \mathcal{P}(S)$ is a Boolean set-assignment such that $S \subseteq O{ }^{10}$

${ }^{10}$ In this notation we use different letters to denote set-assignments because, unlike the models for Epstein's logics, in the models for certain modal logics $o$ is an auxiliary function via which the $s$ function is defined. 
Let $\mathfrak{M}=\langle v, o, s\rangle$ be an m-model. The formula $A \in \mathcal{L}_{\mathrm{M}}$ is true in $\mathfrak{M}$ (in symb.: $\mathfrak{M} \models A$ ) iff for any $B, C \in \mathcal{L}_{\mathrm{M}}$ :

$$
\begin{array}{ll}
v(A)=1, & \text { if } A \in \operatorname{Var}, \\
\text { not } \mathfrak{M} \models B \quad(\text { in symb.: } \mathfrak{M} \not \models B), & \text { if } A=\neg B, \\
\mathfrak{M} \models B \text { and } \mathfrak{M} \models C, & \text { if } A=B \wedge C, \\
\mathfrak{M} \models B \text { and } s(B)=S, & \text { if } A=\square B .
\end{array}
$$

In the case where Epstein defines modal logics in the $\mathcal{L}_{\mathrm{E}}$ language the implication is understood as in the case of the logic DD, i.e.:

$$
\langle v, o, s\rangle \models A \rightarrow B \text { iff }\langle v, o, s\rangle \models A \supset B \text { and } s(A) \subseteq s(B) .
$$

Using the abbreviation we have just introduced, we get:

$$
\langle v, o, s\rangle \models \square A \text { iff }\langle v, o, s\rangle \models A \text { and } s(\neg A) \subseteq s(A) .
$$

Of significance here is that in the case of Boolean set-assignment $s$ we have:

$$
s(A) \subseteq s(B) \text { iff } s(A) \cap s(\neg B)=\emptyset .
$$

With the condition $s(A) \cap s(\neg B)=\emptyset$ Epstein intends to say that the contents of $A$ and $\neg B$ are mutually exclusive. Hence, by claiming that $A$ is necessary, we say that the contents of $A$ and $\neg A$ are mutually exclusive, and by claiming that $A$ is possible, we say that contents of $A$ and $\neg A$ are not mutually exclusive. In other words, a necessary proposition excludes its negation in terms of content, whereas a possible proposition does not exclude its negation in terms of content.

The definition of truth in an m-model adopted by Epstein allows one to prove the following fact:

FACT 2.26 (Epstein, 1990, p. 158, Lemma 2). Let $\mathfrak{M}=\langle v, o, s\rangle$ be an m-model. Then, for any $A, B \in \mathcal{L}_{M}$ :

1. if $A$ is a classical tautology in $\mathcal{L}_{M}$, then $\mathfrak{M} \models A$ and $s(A)=S$, i.e., $\mathfrak{M}=\square A$,

2. $\mathfrak{M} \models \square(A \supset B) \supset(\square A \supset \square B)$,

3. $\mathfrak{M} \models \square A \supset A$.

As a result, we can see that the modal axioms $(\mathrm{K})$ and $(\mathrm{T})$ are always true in an m-model and that any classical tautology must also be true. 
Let us now move on to defining m-models for logics $\mathbf{S} 4$ and $\mathbf{T}$. In what follows we will make reference to the standard understanding of theorem of normal modal logic and to Kripke's models for normal modal logics. However, instead of valuations usually adopted in Kripke's model, like Epstein we will use valuations in the form of $V: W \longrightarrow \mathcal{P}(\operatorname{Var})$. This change does not influence the truth of the theorem on soundness and completeness for modal logics that can be expressed using Kripke's models.

By an m-model for $\mathbf{S} 4$ we will mean an m-model $\langle v, o, s\rangle$ such that $o=s$ and that $s$ satisfies the following conditions:

$$
\begin{aligned}
& s(\square(A \supset B)) \subseteq s(\square A \supset \square B), \\
& s(A)=S \Rightarrow s(\square A)=S, \\
& s(\square A) \subseteq s(A), \\
& s(\square A) \subseteq s(\square \square A) .
\end{aligned}
$$

Any m-model for $\mathbf{S} 4\langle v, o, s\rangle$ may be reduced to an ordered pair $\langle v, s\rangle$.

Epstein proves the following theorem on soundness for the logic S4:

Theorem 2.27 (Epstein, 1990, p. 166, Lemma 6). For any $A \in \mathcal{L}_{M}$, if $A$ is a theorem of $\mathbf{S 4}$, then for any m-model for $\mathbf{S} \mathbf{4}\langle v, s\rangle,\langle v, s\rangle \models A$, and $s(A)=S$.

In order to prove the theorem on completeness, Epstein determines the way in which an m-model is defined with respect to Kripke's model.

Let $\langle W, Q, V\rangle$ be a Kripke's model and let $w \in W$. Then an m-model generated by $\langle W, Q, V\rangle$ and $w$ refers to an ordered pair $\langle v, s\rangle$ such that:

- $S:=\{u \in W: Q(w, u)\}$;

- for any $A \in \operatorname{Var}$ :

$$
v(A)=1 \text { iff } A \in V(w)
$$

- for any $A \in \mathcal{L}_{\mathrm{M}}$ :

$$
s(A):=\{u \in W: Q(w, u) \text { and }\langle W, Q, V\rangle, u \models A\} .
$$

Epstein also proves the following lemma:

Lemma 2.28 (Epstein, 1990, p. 166, Lemma 7a). Let $\langle W, Q, V\rangle$ be a reflexive and transitive Kripke's model and $w \in W$. If $\langle v, s\rangle$ is an $m$ model generated by $\langle W, Q, V\rangle$ and $w$, then:

1. $\langle v, s\rangle$ is an m-model for $\mathbf{S} 4$,

2. for any $A \in \mathcal{L}_{M},\langle v, s\rangle \models A$ iff $\langle W, Q, V\rangle, w \models A$. 
By lemma 2.28 and the standard theorem on completeness, using reflexive and transitive Kripke's models, it is possible to obtain the following theorem on completeness for the logic S4:

Theorem 2.29 (Epstein, 1990, p. 166, Lemma 7b). For any $A \in \mathcal{L}_{M}$, if $A$ is not a theorem of $\mathbf{S} 4$, then there exists an m-model for $\mathbf{S} 4\langle v, s\rangle$ such that $\langle v, s\rangle \forall A$ and $s(A) \neq S$.

Let us now define m-models for the normal modal logic T. By an $m$-model for $\mathbf{T}$ we mean an m-model $\langle v, o, s\rangle$ such that $s(A):=o(A) \cap S$ and $o$ satisfies the following conditions:

$$
\begin{aligned}
& o(\square(A \supset B)) \subseteq o(\square A \supset \square B), \\
& o(A)=O \Rightarrow o(\square A)=O, \\
& o(\square A) \subseteq o(A) .
\end{aligned}
$$

Note that if $o: \mathcal{L}_{\mathrm{M}} \longrightarrow \mathcal{P}(O)$ is a Boolean set-assignment then $s: \mathcal{L}_{\mathrm{M}} \longrightarrow$ $\mathcal{P}(S)$ such that $s(A):=o(A) \cap S$ is also a Boolean set-assignment. Therefore, $\langle v, o, s\rangle$ is indeed an m-model.

The following theorem on the soundness for the logic $\mathbf{T}$ is proved:

Theorem 2.30 (Epstein, 1990, p. 173, Lemma 18). If $A$ is a theorem of $\mathbf{T}$, then for any m-model for $\mathbf{T}\langle v, o, s\rangle,\langle v, o, s\rangle \models A$, and $s(A)=S$.

Epstein modifies the above method of defining an m-model with regards to Kripke's model. Let $\langle W, Q, V\rangle$ be a Kripke's model and $w \in W$, a modified m-model generated by $\langle W, Q, V\rangle$ and $w$ refers to an ordered triple $\langle v, o, s\rangle$ in which:

- $O=W$,

- $S:=\{u \in W: Q(w, u)\}$,

- for any $A \in$ Var:

$$
v(A)=1 \text { iff } A \in V(w),
$$

- for any $A \in \mathcal{L}_{\mathrm{M}}$ :

$$
\begin{aligned}
& o(A):=\{u \in W:\langle W, Q, V\rangle, u \models A\}, \\
& s(A):=o(A) \cap S .
\end{aligned}
$$

The following lemma is also proven within Epstein's work:

Lemma 2.31 (Epstein, 1990, p. 173, Theorem 19). Let $\langle W, Q, V\rangle$ be a reflexive Kripke's model and $w \in W$. If $\langle v, o, s\rangle$ is a modified m-model generated by $\langle W, Q, V\rangle$ and $w$, then: 
1. $\langle v, o, s\rangle$ is an m-model for $\mathbf{T}$,

2. for any $A \in \mathcal{L}_{M},\langle v, o, s\rangle \models A$ if $\langle W, Q, V\rangle, w \models A$.

By the above lemma, Epstein proves the following theorem on completeness:

Theorem 2.32 (Epstein, 1990, p. 173, Theorem 19). For any $A \in \mathcal{L}_{M}$, if $A$ is not a theorem of $\mathbf{T}$, then there exists an m-model for $\mathbf{T}\langle v, o, s\rangle$ such that $\langle v, o, s\rangle \not \models A$ and $s(A) \neq S$.

The final element considered within this section is the set-assignment semantics defined for the logic K. Like before, Epstein makes use of mmodels; however, he modifies the definition of a truth in the model by assuming the following condition for modal formulae:

$$
\langle v, o, s\rangle \models \square A \text { iff } s(A)=S .
$$

In this case a question arises as to whether this interpretation is logically correct. From the syntactic perspective, modal operators are unary proposition-creating functors. However, in line with the proposed interpretation, modality is a certain type of predicate. Therefore, in these circumstances we are dealing with a certain category error. Of course, one can eliminate this problem by assuming a predicative nature of modality, but then one must also exclude any cases of modality iteration from the modal language. This, in turn, excludes an analysis of modal logics understood in a standard way.

\section{The Torunian Programme of Relating Semantics}

The Torunian Programme of Relating Semantics may be stated as follows [cf. Jarmużek, 2021; Jarmużek and Kaczkowski, 2014]:

1. Main assumption: there are two sentence properties that are significant for logical research: logical values of sentences (the extensional aspect of logical research) and the connection of sentences in terms of relationships that occur between that what these sentences refer to (the non-extensional aspect of logical research).

2. Main goals: to introduce new logics motivated by various properties because of which we may consider sentences to be related with each other and the semantic analysis of known non-classical logics based on the main assumption (see problems $\alpha-\eta$ ). 
3. Main assumption of relating logics: the logical value of compound sentences constructed using various connectives depends on:

- the logical values of component sentences,

- any form of relationship due to which the component sentences may be considered to be related one to another.

At this point, it is worth noting that unlike Epstein's Programme the Torunian Programme advocates that all types of relationships by which it may be said that certain sentences are related should be taken into account. This translates to a huge variety of interpretations of connectives. In particular, just as Epstein did, one may account for all sorts of content relationships. However, there are also other relationships:

1. objective relationships, such as:

- temporal relationships, expressed in the following example: John met Barbara and married her;

- causal relationships, expressed in the following example: if you switch off the light, the room will be dark.

2. subjective relationships, such as:

- preference, expressed in the following example: Barbara drinks coffee or tea, which she prefers; if John cleans the room, he will go to the movies, although he would have preferred to only go to the movies;

- justified beliefs, expressed in the following example: the result was negative and that's why, according to John, Peter is alright.

3. normative relationships, such as:

- lawfulness of conduct, expressed in the following example: if John built a garage, he must have had a permit;

- morality of conduct, expressed in the following example: if John helped Peter, he did the right thing.

Insights which were similar, at least to some extent, were presented by Walton [1979a], who highlighted the possibility of using the relatedness relation to analyse various relationships. However, in many cases the assumption of reflexivity which is assumed in the case of the relatedness relation will be at least non-intuitive. Moreover, the Torunian Programme postulates not only an analysis of already existing philosophical propositional logics (relevance, temporal, causal, preference, epistemic, or deontic), but first and foremost it advocates the introduction of some completely new logics of this kind. In the following section we will describe selected results which exemplify the implementation of this postulate. 


\subsection{A general approach to relating logic and semantics}

Before we move on to describe the fundamental problems of relating logic, we will present a general description referring to the approach proposed by Jarmużek [2021]. To this end, let us consider a propositional language, defined for any $m, n \in \mathbb{N}$ :

$$
\mathcal{L}_{m, n}:=\left\langle\operatorname{Var},\left\{\pi_{i}: 1 \leqslant i \leqslant m\right\},\left\{\rho_{i}: 1 \leqslant i \leqslant n\right\},\{),(\}\right\rangle,
$$

where:

- $\left\{\pi_{i}: 1 \leqslant i \leqslant m\right\}$ and $\left\{\rho_{i}: 1 \leqslant i \leqslant n\right\}$ are sets of logical connectives,

- $\left\{\pi_{i}: 1 \leqslant i \leqslant m\right\} \cap\left\{\rho_{i}: 1 \leqslant i \leqslant n\right\}=\emptyset$,

- $\left\{\pi_{i}: 1 \leqslant i \leqslant m\right\} \cup\left\{\rho_{i}: 1 \leqslant i \leqslant n\right\} \neq \emptyset$ (tj. $m \neq 0$ lub $n \neq 0$ ).

The set of $\mathcal{L}_{m, n}$ formulae is defined in a standard way and will be denoted by $\mathcal{L}_{m, n}$. This language is a general propositional language. The first of the sets of connectives includes those connectives which we will interpret extensionally, whereas the second includes those which we will interpret in a relating manner. Therefore, these sets must be disjoint. As can be seen, we also assume that at least one of these sets is non-empty.

Below, we will define the relating semantics and logic in a general manner. This approach will be an interpretation of the language $\mathcal{L}_{m, n}$.

The first element of relating semantics is the valuation of propositional variables, i.e., a function $v: \operatorname{Var} \longrightarrow L V$ whereby $L V$ is a set of values whose power is greater than or equal to 2 .

For any $1 \leqslant i \leqslant m$ and $1 \leqslant j \leqslant n$, we define the truth functions: $f_{\pi_{i}}: L V^{\operatorname{ar}\left(\pi_{i}\right)} \longrightarrow L V$ and $f_{\rho_{j}}: L V^{\operatorname{ar}\left(\rho_{j}\right)} \longrightarrow L V$. These functions express the meaning of connectives indexed $i$ and $j$, respectively.

The second element of relating semantics is the connection evaluation, i.e., a function $e_{i}: \mathcal{L}_{m, n}^{\operatorname{ar}\left(\rho_{i}\right)} \longrightarrow C L V$ in which $C L V$ is the set of connection (logical) values whose power is greater than or equal to 2 .

The third element is the set of distinguished logical values and the set of distinguished connection values: $L V^{+} \subseteq L V$ and $C L V^{+} \subseteq C L V$.

Connection evaluation allows one to formally grasp the connection of sentences in terms of various content or extra-content relationships between that what these sentences refer to. By using the connection values, one can formally represent connections of various types or a gradable connection of a single type.

By a relating model (a relating model of language $\mathcal{L}_{m, n}$ ), we refer to an ordered pair $\left\langle v,\left\{e_{i}\right\}_{1 \leqslant i \leqslant n}\right\rangle$ such that:

- $v: \operatorname{Var} \longrightarrow L V$ is a valuation of propositional variables, 
- $\left\{e_{i}\right\}_{1 \leqslant i \leqslant n}$ is a family of connection evaluations, i.e., for any $1 \leqslant i \leqslant n$, $e_{i}: \mathcal{L}_{m, n}^{\operatorname{ar}\left(\rho_{i}\right)} \longrightarrow C L V$.

Let $\mathfrak{M}$ be a relating model. For any $1 \leqslant i \leqslant m$ and $1 \leqslant j \leqslant n$ the following truth-conditions can be defined:

$$
\begin{array}{r}
\mathfrak{M} \models \pi_{i}\left(A_{1}, \ldots, A_{\operatorname{ar}\left(\pi_{i}\right)}\right) \text { iff } f_{\pi_{i}}\left(V\left(A_{1}\right), \ldots, V\left(A_{\operatorname{ar}\left(\pi_{i}\right)}\right)\right) \in L V^{+}, \\
\mathfrak{M} \models \rho_{j}\left(A_{1}, \ldots, A_{\operatorname{ar}\left(\rho_{j}\right)}\right) \text { iff }\left[f _ { \rho _ { j } } \left(V\left(A_{1}\right), \ldots, V\left(A_{\left.\left.\operatorname{ar}\left(\rho_{j}\right)\right)\right) \in L V^{+}}\right.\right.\right. \\
\text {and } \left.e_{j}\left(A_{1}, \ldots, A_{\operatorname{ar}\left(\rho_{j}\right)}\right) \in C L V^{+}\right] .
\end{array}
$$

A relating model $\left\langle v,\left\{e_{i}\right\}_{1 \leqslant i \leqslant n}\right\rangle$ is called:

- mono-relating iff for any $1 \leqslant i, j \leqslant n, e_{i}=e_{j}$, i.e., the power of $\left\{w_{i}\right\}_{i \leqslant n}$ equals 1 ,

- multi-relating iff there are $1 \leqslant i \neq j \leqslant n$ such that $e_{i} \neq e_{j}$, i.e., the power of $\left\{e_{i}\right\}_{i \leqslant n}$ is greater than 1 .

The relating logic defined using mono-relating (multi-relating) models is known as a mono-relating logic (multi-relating logic).

A model with two logical values and two connection values is a special case of a relating model:

- $L V=C L V:=\{1,0\}$,

- $L V^{+}=C L V^{+}:=\{1\}$.

Any evaluation of connection may then be equated with a finite relation defined on the set of formulae:

$$
R_{i}\left(A_{1}, \ldots, A_{\operatorname{ar}(i)}\right) \text { iff } e_{i}\left(A_{1}, \ldots, A_{\operatorname{ar}(i)}\right)=1 .
$$

The relation $R \subseteq F^{k}$ such that $F$ is the set of formulae and $k \geqslant 1$ is known as the connection relation or the relating relation. A monorelating logic (multi-relating logic) defined using relations is called a mono-relational relating logic (multi-relational relating logic).

\subsection{Research directions and selected results}

In the framework of the Torunian Programme, one can distinguish five general problems which determine the most important directions of research:

- The $\alpha$ problem: to describe formulae and/or principles adequate for logics determined by distinguished relating models and/or connection evaluations (the problem of syntactic characterisation).

- The $\beta$ problem: to describe relating models and/or connection evaluations appropriate for the logics determined by distinguished formulae and/or principles (the problem of semantic characterisation). 
- The $\gamma$ problem: to define philosophical connectives by means of relating connectives; to express new and/or known philosophical logics in terms of relating logic (the problem of relating reduction).

- The $\delta$ problem: to analyse the properties of relating semantics and the connections between the relating semantics and other semantics (the problem of characterisation with regards to relating semantics). - The $\eta$ problem: to connect the relating semantics to other semantics (the problem of characterisation with regards to connected semantics). ${ }^{11}$

Of course, the above problems are not disjoint. For example, $\gamma$ is related to $\alpha$ and $\beta$. By introducing various philosophical logics described using relating semantics, one may enquire about their appropriate axiomatisation as well as other adequate syntactic (proof-theoretic) settings. $\eta$, in turn, is undoubtedly related to $\delta$ and $\alpha$. When studying semantics formed from the connection of some other semantics with the relating semantics it is only natural to ask questions about the connections between the starting semantics and the relating semantics, e.g., if the logics determined by a given semantics can also be determined by the relating semantics. Moreover, as specified earlier, one may consider axiomatisation and other syntactic (proof-theoretic) settings of the logics defined by means of semantics formed via the connection of some given semantics and the relating semantics. In the following analysis, we will describe selected results which contribute to the implementation of the Torunian Programme through their function as special cases of the fundamental problems listed above.

The first work to present relating logics other than Epstein's logics - and consequently extending the founding perspective of Epstein's Programme - is the work by Jarmużek and Kaczkowski [2014]. In this paper they considered the smallest mono-relational relating logic, known as the logic $\mathbf{F}$ and described in the $\mathcal{L}_{\Lambda^{w}, \rightarrow^{w}}$ language with five standard connectives $\neg, \wedge, \vee, \rightarrow, \leftrightarrow$ and two relating connectives $\wedge^{w}$ (relating conjunction) and $\rightarrow^{w}$ (relating implication). ${ }^{12}$ The language $\mathcal{L}_{\Lambda^{w}, \rightarrow^{w}}$ is a special case of the language $\mathcal{L}_{5,2}$. The set of formulae in $\mathcal{L}_{\wedge^{w}, \rightarrow^{w}}$ is defined in a standard way and is denoted by $\mathcal{L}_{\wedge^{\mathrm{w}}, \rightarrow^{\mathrm{w}}}$. A model of $\mathcal{L}_{\wedge^{\mathrm{w}}, \rightarrow}$

11 These general problems were officially announced in the call for the 1st Workshop On Relating Logic, September 25-26, 2020, https://www.filozofia.umk.pl/ en/department-of-logic/call-for-workshop-on-relating-logic/

12 Jarmużek and Kaczkowski used a different notation and denoted the relating conjunction and relating implication by $\triangle$ and $\leftrightarrow$, respectively. 
consists of a unique version of a mono-relational relating model, i.e., an ordered pair of the form $\langle v, R\rangle$, where $R$ is a binary relation over $\mathcal{L}_{\wedge^{w}, \rightarrow^{w}}$. Since the logic $\mathbf{F}$ had been described semantically, with the appropriate tableau systems provided on a subsequent basis, one can say that the paper produced by Jarmużek and Kaczkowski contributes to analysis of the problem $\alpha$. The work also suggests possible further research exploring $\gamma$ and $\delta$ problems.

Unlike the analysis carried out by Epstein, Jarmużek and Kaczkowski's paper is not about describing two or four logics for a given connection; rather, it is about describing an entire family of logics of a certain type, the analysis of which begins by the smallest logic of this given type. Jarmużek and Kaczkowski demonstrate that a relating logic should be seen as a certain family of logics. Assuming a language, the starting point for the analysis of a given family of relating logics would be the smallest logic defined by all the models of a given language. Using normal modal logics as an example, we might venture making the following comparison: Epstein takes the perspective of someone who is interested in two or four modal systems because of this or that application of these systems, whereas Jarmużek and Kaczkowski attempt to employ the perspective of someone who is asking about an entire family of normal modal logics and who begins to analyse it using the most basic system, i.e., the normal modal logic $\mathbf{K}$.

A similar approach can be observed in the work of Jarmużek and Malinowski [2019a], where relating semantics is used to define a family of Boolean connexive logics (for short: BCL). The language of BCL, i.e., the language $\mathcal{L}_{\mathrm{BCL}}$, is a special case of the language $\mathcal{L}_{3,1}$. It includes three Boolean connectives $\neg, \wedge, \vee$ and also implication $\rightarrow$. In a similar manner to earlier discussions, the set of formulae in $\mathcal{L}_{\mathrm{BCL}}$ is defined in a standard way and is denoted by $\mathcal{L}_{\mathrm{BCL}}$. A model of $\mathcal{L}_{\mathrm{BCL}}$, just as in the case of the logic $\mathbf{F}$, consists of an ordered pair of the form $\langle v, R\rangle$. This time, however, $R \subseteq \mathcal{L}_{\mathrm{BCL}} \times \mathcal{L}_{\mathrm{BCL}}$ is a binary relation that satisfies the following conditions:

$$
\begin{aligned}
& \forall_{A \in \mathcal{L}_{\mathrm{BCL}}} \sim R(A, \neg A), \\
& \forall_{A \in \mathcal{L}_{\mathrm{BCL}}} \sim R(\neg A, A), \\
& \forall_{A, B \in \mathcal{L}_{\mathrm{BCL}}}(R(A, B) \Rightarrow \sim R(A, \neg B)), \\
& \forall_{A, B \in \mathcal{L}_{\mathrm{BCL}}} R(A \rightarrow B, \neg(A \rightarrow \neg B)), \\
& \forall_{A, B \in \mathcal{L}_{\mathrm{BCL}}} R(A \rightarrow \neg B, \neg(A \rightarrow B)) .
\end{aligned}
$$


By Aristotle's relation we mean any binary relation over $\mathcal{L}_{\mathrm{BCL}}$ that satisfies (a1) and (a2), and by Boethius' relation we mean any binary relation over $\mathcal{L}_{\mathrm{BCL}}$ that satisfies (b1)-(b3).

The first three $\mathcal{L}_{\mathrm{BCL}}$ connectives, i.e., $\neg, \wedge, \vee$, are interpreted classically. In turn, the implication $\rightarrow$ is interpreted in a relating manner as follows:

$$
\langle v, R\rangle \models A \rightarrow B \text { iff }(\langle v, R\rangle \not \models A \text { or }\langle v, R\rangle \models B \text {, and } R(A, B)) \text {. }
$$

The conditions for a connexive relation ensure that the connexive theorems are true. These theorems are attributed to Aristotle and Boethius, respectively:

$$
\begin{aligned}
& \text { Aristotle's theorems }\left\{\begin{array}{l}
\neg(A \rightarrow \neg A) \\
\neg(\neg A \rightarrow A)
\end{array}\right. \\
& \text { Boethius' theorems }\left\{\begin{array}{l}
(A \rightarrow B) \rightarrow \neg(A \rightarrow \neg B) \\
(A \rightarrow \neg B) \rightarrow \neg(A \rightarrow B) .
\end{array}\right.
\end{aligned}
$$

Aristotle's (Boethius') relations ensure the validity of Aristotle's (Boethius') theorems.

In [Jarmużek and Malinowski, 2019a] the BCLs were defined semantically, with the complete tableau systems defined in turn. Consequently, the work falls within the scope of the problem $\alpha$. Moreover, it also falls within the scope of the problem $\gamma$ because the connexive implication is expressed using the relating implication. The situation is similar in [Jarmużek and Malinowski, 2019b], where the modal Boolean connexive logics (for short: MBCL) are described semantically and the complete tableau systems for the logics under study are provided. In [Klonowski, 2021] for some basic and modal Boolean connexive logics complete axiomatic systems were presented. Apart from the above works, BCLs have also been analysed by Malinowski and Palczewski [2021]. In this work, the possibility of using BCL in the analysis of Lewis Carroll's barber paradox is presented.

A connexive logic might be characterised syntactically as a set of formulae that includes Aristotle's and Boethius' theorems, which is closed under substitution and Modus Pones and that does not include a law of symmetry of implication: $(A \rightarrow B) \rightarrow(B \rightarrow A)$ [see Jarmużek and Malinowski, 2019a; Wansing, 2020]. Hence, BCL may be characterised syntactically as a connexive logic that includes Boolean logic, i.e., the 
classical logic described with connectives $\neg, \wedge, \vee$ and the following arbitrary formula: $\neg(A \rightarrow B) \vee(\neg A \vee B)$ (this formula states that the connexive implication strengthens the material implication [see Klonowski, 2021]. This starting point allows one to consider various BCLs in syntactic setting, i.e., as postulated in $\beta$.

Note that the work [Jarmużek and Malinowski, 2019b] also falls within the scope of the problem $\eta$ as it provides a peculiar connection of semantics of possible worlds with the relating semantics. The situation is similar in [Jarmużek and Klonowski, 2020], where connected Kripke's models are used together with relating models, this time, however, to define some kind of deontic logic. In this work the problem of relevance of deontic sentences is accounted for from the point of view of a given normative system, understood as a set of certain principles (imperatives and prohibitions).

In order to express a given setting let us consider a deontic language $\mathcal{L}_{\mathrm{D}}$, a special case of the $\mathcal{L}_{5,2}$ language which includes the standard connectives $\neg, \wedge, \vee, \rightarrow, \leftrightarrow$ and the deontic operators $\mathrm{O}, \mathrm{P}$. The set of formulae in this language is defined in a standard way and is denoted by $\mathcal{L}_{\mathrm{D}}$. The model of $\mathcal{L}_{\mathrm{D}}$ has the form of an ordered quadruple $\left\langle W, Q,\left\{R_{w}\right\}_{w \in W}, V\right\rangle$, where $\langle W, Q, V\rangle$ is a Kripke's model and $\left\{R_{w}\right\}_{w \in W}$ is a family of connection relations, i.e., for any world $w$ we introduce a relation with a fixed arity $n R_{w} \subseteq \mathcal{L}_{\mathrm{D}}^{n} \cdot{ }^{13}$ For simplicity we consider models with a family of unary connection relations, i.e., models with a family of subsets of the set of formulae $\mathcal{L}_{\mathrm{D}} \cdot{ }^{14}$ The standard connectives have a classical interpretation, while the deontic operators are interpreted as follows:

$$
\begin{aligned}
& \mathfrak{M}, w \models \mathrm{O} A \text { iff } \forall_{u \in W}\left(Q(w, u) \Rightarrow\left(\mathfrak{M}, u \models A \text { and } A \in R_{u}\right)\right), \\
& \mathfrak{M}, w \models \mathrm{P} A \text { iff } \exists_{u \in W}\left(Q(w, u) \text { and }\left(\mathfrak{M}, u \not \models A \text { or } A \notin R_{u}\right)\right) .
\end{aligned}
$$

One can see that in the stated truth-conditions one accounts for not only the logical value of the component sentence in the accessible deontic alternatives but also for the question of whether a given sentence is relevant from the point of view of a given normative system, in a given alternative. To some extent, this approach is similar to that of Fagin and

13 In [Jarmużek and Malinowski, 2019b] a family of binary connection relations has been used; similarly in [Jarmużek and Klonowski, 2020].

14 In [Jarmużek and Klonowski, 2020] the subsets of the set of formulae $\mathcal{L}_{\mathrm{D}}$ are defined using binary connection relations. 
Halpern [1988], which is based on the so-called awareness function. What differs the former from the latter is that there is no functor counterpart of the connection relation in the object language and the connection relations matter not only in the actual world but also in any accessible world.

The work [Jarmużek and Klonowski, 2020] falls not only within the scope of the problem $\eta$ but also within the scope of the problem $\alpha$, as the deontic logics in question have been defined semantically (followed by the complete tableau systems).

Yet another example of a work that falls within problem $\alpha$ is the study by Jarmużek and Klonowski [2021], which presents an analysis of tableau systems for selected classical mono-relating logics (for short: CMRL). In fact, CMRL are a kind of basis for the mono-relational relating logics considered so far - not only by the Toruń Logic Group but also by other logicians. The CMRL language, i.e., the language $\mathcal{L}_{\mathrm{CMRL}}$ is a special case of the language $\mathcal{L}_{5,4}$. It includes five standard connectives $\neg, \wedge, \vee, \rightarrow, \leftrightarrow$ and four relating connectives: $\wedge^{w}, \vee^{w}, \rightarrow^{w}, \leftrightarrow^{w}$. In other words, $\mathcal{L}_{\mathrm{CMRL}}$ is an extension of $\mathcal{L}_{\wedge^{\mathrm{w}}, \rightarrow^{\mathrm{w}}}$ through the use of two relating connectives: $V^{w}$ and $\leftrightarrow^{w}$. The set of formulae of $\mathcal{L}_{\mathrm{CMRL}}$ is defined in a standard way and is denoted by $\mathcal{L}_{\mathrm{CMRL}}$.

The logics under analysis in [Jarmużek and Klonowski, 2021] are motivated by selected causal relation properties; moreover, they are defined by means of relating models in the form of $\langle v, R\rangle$, where $R \subseteq$ $\mathcal{L}_{\mathrm{CMRL}} \times \mathcal{L}_{\mathrm{CMRL}}$ is a binary relation satisfying at least one of the following conditions:

$$
\begin{aligned}
& \forall_{A \in \mathcal{L}_{\mathrm{CMRL}}} \sim R(A, A), \\
& \forall_{A, B \in \mathcal{L}_{\mathrm{CMRL}}}(R(A, B) \Rightarrow \sim R(B, A)), \\
& \forall_{A, B, C \in \mathcal{L}_{\mathrm{CMRL}}}((R(A, B) \text { and } R(B, C)) \Rightarrow R(A, C)), \\
& \forall_{A, B \in \mathcal{L}_{\mathrm{CMRL}}}(R(\neg A, B) \Rightarrow R(A, B)), \\
& \forall_{A, B \in \mathcal{L}_{\mathrm{CMRL}}}(R(A, \neg B) \Rightarrow R(A, B)), \\
& \forall_{A, B, C \in \mathcal{L}_{\mathrm{CMRL}}}(R(A \wedge B, C) \Rightarrow(R(A, C) \text { or } R(B, C))), \\
& \forall_{A, B, C \in \mathcal{L}_{\mathrm{CMRL}}}(R(A, B \wedge C) \Rightarrow(R(A, B) \text { or } R(A, C))) .
\end{aligned}
$$

Conditions (Ir), (As), and (Tr) are the well-known relational conditions of irreflexivity, asymmetry, and transitivity. In turn, $\left(\mathrm{E}_{\neg 1}\right),\left(\mathrm{E}_{\neg 2}\right),\left(\mathrm{E}_{\mathrm{or}}^{\wedge_{1}}\right)$, and $\left(\mathrm{E}_{\mathrm{or}}^{\wedge}\right)$ are the conditions of elimination of negation and conjunction from the first and the second argument of the relation, respectively. 
A detailed CMRL analysis is presented in [Jarmużek and Klonowski, submitted; Klonowski, 2019]. In this work, selected meta-logical properties of CMRL are analysed such as the expressiveness of relating connectives, the ability to define the connection relation, the proof of compactness by the semantic method, as well as the proof of completeness with regards to axiomatic systems. Consequently, the work falls within the scope of problems $\alpha$ and $\delta$. The work in question also analyses the logics described by models with connection relations which satisfy at least one horizontal and/or vertical condition. By a horizontal condition we mean a condition in which we do not make reference to the way the formula is constructed. A vertical condition, however, is a condition for which the way a formula is constructed is important.

An example of a horizontal condition is provided by the irreflexivity (Ir), asymmetry (As), or transitivity (Tr), as well as the following conditions accounted for in [Klonowski, 2019]:

$$
\begin{aligned}
& \forall_{A \in \mathcal{L}_{\mathrm{CMRL}}} R(A, A), \\
& \forall_{A, B \in \mathcal{L}_{\mathrm{CMRL}}}(R(A, B) \Rightarrow R(B, A)), \\
& \forall_{A, B \in \mathcal{L}_{\mathrm{CMRL}}}(R(A, B) \text { or } R(B, A)), \\
& \forall_{A, B \in \mathcal{L}_{\mathrm{CMRL}}}(A \neq B \Rightarrow(\sim R(A, B) \text { or } \sim R(B, A))) .
\end{aligned}
$$

Condition ( $\mathrm{Re})$ is the condition of reflexivity, (Sym) is the condition of symmetry, (Co) is the condition of connexity, and ( $\mathrm{An}$ ) is the condition of asymmetry. Other important horizontal conditions include the following two conditions:

$$
\begin{array}{rl}
\forall_{A, B \in \mathcal{L}_{\mathrm{CMRL}}} & R(A, B), \\
\forall_{A, B \in \mathcal{L}_{\mathrm{CMRL}}} \sim R(A, B) .
\end{array}
$$

These conditions characterise the universal relation and the empty relation, respectively. ${ }^{15}$ Clearly, a logic determined by relating models with the universal relation corresponds to classical propositional logic.

Examples of vertical conditions include $\left(\mathrm{E}_{\neg 1}\right),\left(\mathrm{E}_{\neg 2}\right),\left(\mathrm{E}_{\mathrm{or}}^{\wedge_{1}}\right)$, and $\left(\mathrm{E}_{\mathrm{or}}^{\wedge_{2}}\right)$. Moreover, in [Klonowski, 2019] the following vertical conditions were considered:

- $\quad$ an introduction of negation $\neg$ :

$$
\begin{aligned}
& \forall_{A, B \in \mathcal{L}_{\mathrm{CMRL}}}(R(A, B) \Rightarrow R(\neg A, B)), \\
& \forall_{A, B \in \mathcal{L}_{\mathrm{CMRL}}}(R(A, B) \Rightarrow R(A, \neg B)) ;
\end{aligned}
$$

15 Note that the relating models based on the universal relation (the models based on the empty relation) only differ in their valuation. 
- an elimination of the binary connective $* \in\left\{\wedge, \vee, \rightarrow, \leftrightarrow, \wedge^{\mathrm{w}}, \vee^{\mathrm{w}}, \rightarrow^{\mathrm{w}}\right.$, $\left.\leftrightarrow^{\mathrm{w}}\right\}$ :

$\forall_{A, B, C \in \mathcal{L}_{\mathrm{CMRL}}}(R(A * B, C) \Rightarrow(R(A, C)$ or $R(B, C)))$,

$\forall A, B, C \in \mathcal{L}_{\mathrm{CMRL}}(R(A, B * C) \Rightarrow(R(A, B)$ or $R(A, C)))$.

$\forall_{A, B, C \in \mathcal{L}_{\mathrm{CMRL}}}(R(A * B, C) \Rightarrow(R(A, C)$ and $R(B, C)))$,

$\left(\mathrm{E}_{\text {and }}^{*_{1}}\right)$

$\forall_{A, B, C \in \mathcal{L}_{\mathrm{CMRL}}}(R(A, B * C) \Rightarrow(R(A, B)$ and $R(A, C)))$;

$\left(\mathrm{E}_{\text {and }}^{*_{2}}\right)$

- $\quad$ an introduction of the binary connective $* \in\left\{\wedge, \vee, \rightarrow, \leftrightarrow, \wedge^{\mathrm{w}}, \vee^{\mathrm{w}}, \rightarrow^{\mathrm{w}}\right.$, $\left.\leftrightarrow^{w}\right\}$ :

$$
\begin{aligned}
& \forall_{A, B, C \in \mathcal{L}_{\mathrm{CMRL}}}((R(A, C) \text { or } R(B, C)) \Rightarrow R(A * B, C)), \quad\left(\mathrm{I}_{*_{1}}^{\text {or }}\right) \\
& \forall A, B, C \in \mathcal{L}_{\mathrm{CMRL}}((R(A, C) \text { or } R(B, C)) \Rightarrow R(A, B * C)), \quad\left(\mathrm{I}_{*_{2}}^{\text {or }}\right) \\
& \forall A, B, C \in \mathcal{L}_{\mathrm{CMRL}}((R(A, C) \text { and } R(B, C)) \Rightarrow R(A * B, C)), \quad\left(\mathrm{I}_{*_{1}}^{\text {and }}\right) \\
& \forall_{A, B, C \in \mathcal{L}_{\mathrm{CMRL}}}((R(A, B) \text { and } R(A, C)) \Rightarrow R(A, B * C)) . \quad\left(\mathrm{I}_{*_{2}}^{\text {and }}\right)
\end{aligned}
$$

These conditions are a weakened version of Epstein's conditions and, together with appropriate horizontal conditions, they allow an expression of the counterparts of the relatedness logics $\mathbf{R}, \mathbf{S}$ and of the dependence $\operatorname{logics} \mathbf{D}, \mathbf{D D}$ in CMRL. We can define:

- $\quad$ r-relations over formulas of $\mathcal{L}_{\mathrm{CMRL}}$ by $(\mathrm{Re}),\left(\mathrm{E}_{\neg 1}\right),\left(\mathrm{I}_{\neg_{1}}\right),\left(\mathrm{E}_{\neg 2}\right),\left(\mathrm{I}_{\neg_{1}}\right)$, $\left(\mathrm{E}_{\mathrm{or}}^{*_{1}}\right),\left(\mathrm{I}_{*_{1}}^{\mathrm{Or}}\right),\left(\mathrm{E}_{\mathrm{or}}^{*_{2}}\right),\left(\mathrm{I}_{*_{2}}^{\mathrm{or}}\right)$;

- sr-relations over formulas of $\mathcal{L}_{\mathrm{CMRL}}$ by $(\mathrm{Re}),(\mathrm{Sym}),\left(\mathrm{E}_{\neg 1}\right),\left(\mathrm{I}_{\neg_{1}}\right)$, $\left(\mathrm{E}_{\mathrm{or}}^{* 1}\right),\left(\mathrm{I}_{*_{1}}^{\mathrm{or}}\right)$;

- d-relations over formulas of $\mathcal{L}_{\mathrm{CMRL}}$ by $(\mathrm{Re}),(\operatorname{Tr}),\left(\mathrm{E}_{\neg 1}\right),\left(\mathrm{I}_{\neg_{1}}\right),\left(\mathrm{E}_{\neg 2}\right)$, $\left(\mathrm{I}_{\neg_{2}}\right),\left(\mathrm{E}_{\text {and }}^{*_{2}}\right),\left(\mathrm{I}_{*_{2}}^{\text {and }}\right)$;

- dd-relations over formulas of $\mathcal{L}_{\mathrm{CMRL}}$ by $(\operatorname{Re}),(\operatorname{Tr}),\left(\mathrm{E}_{\neg 1}\right),\left(\mathrm{I}_{\neg_{1}}\right)$, $\left(\mathrm{E}_{\neg 2}\right),\left(\mathrm{I}_{\neg_{2}}\right),\left(\mathrm{E}_{\text {and }}^{*_{1}}\right),\left(\mathrm{I}_{*_{1}}^{\text {and }}\right)$.

Lastly, let us consider once more the definitions of epistemic operators using the relatedness implication proposed by Jarmużek [2021]. This issue falls within the scope of the problem $\gamma$. The knowledge operator $\mathrm{K}$ is defined as follows:

$$
\mathrm{K} A:=\top \rightarrow^{\mathrm{w}} A .
$$

Expression $T \rightarrow^{\mathrm{w}} A$ may be understood as follows: $A$ has at least one justification which is as good as the best justified statement. By $T$, Jarmużek understands the standards of knowledge and the knowledge 
itself is presented classically. In this setting to know that $A$ means the same as to have a justification for $A$ that is no worse in comparison to that given for the standard of knowledge, which corresponds with the classical definition of knowledge. Moreover, since the standards of knowledge are true sentences in every model, the knowledge must imply truth (as required by the classical setting). The belief operator B is defined as follows:

$$
\mathrm{B} A:=A \rightarrow^{\mathrm{w}} \perp .
$$

In this case it is stated that to believe in $A$ means the same as to have a justification for $A$ that is no worse in comparison to that given for the worst-justified statement. By $\perp$ Jarmużek understands the standard of ignorance, i.e., a sentence that is false in every model.

\section{Summary - comparison of programmes}

Let us now compare the two presented programmes (see Table 2). Firstly, while Epstein's Programme postulates that content relationship should be accounted for in logical research, the Torunian Programme assets that any relationship matters if said relationship encourages the conclusion that the two sentences are connected. In order to define his logics, Epstein adopts at least reflexive relations over the set of formulas which additionally satisfy certain specific conditions. Nonetheless, it is clear that they are special cases of connection relations. Hence, Epstein's logics are the special cases of relating logics postulated by the Torunian Programme.

In the monograph [Epstein, 1990] general relational models are described of the form $\left\langle v, R_{1}, \ldots R_{n}\right\rangle$, where $v$ is a classical valuation of propositional variables and for any $i \leqslant n, R_{i}$ is a connection relation. As shown above, such models are special relating models of the form $\left\langle v,\left\{e_{i}\right\}_{1 \leqslant i \leqslant n}\right\rangle$. Obviously, in the case of general relating models neither valuation nor connection evaluation are required to have a two-valued range.

The main logical research tool used in the framework of Epstein's Programme is set-assignment. Both the content relationship logics (Epstein's logic), and the already known, distinguished, non-classical logics are described by Epstein [1990] [cf. Krajewski, 1991] via functional semantics based on set-assignments, i.e., by using set-assignments semantics. 


\begin{tabular}{|c|c|c|}
\hline & Epstein's approach & Torunian approach \\
\hline relationships & $\begin{array}{l}\text { mainly content (relevance, } \\
\text { analyticity) }\end{array}$ & $\begin{array}{l}\text { content, causal, temporal, } \\
\text { normative, etc. }\end{array}$ \\
\hline relations & at least reflexive & any kind \\
\hline $\begin{array}{l}\text { general } \\
\text { relating } \\
\text { models }\end{array}$ & $\begin{array}{l}\text { relations of formulae } \\
\text { of any arity } \\
+ \\
\text { two logical values }\end{array}$ & $\begin{array}{l}\text { evaluations of } \\
\text { connections } \\
+ \\
\text { many logical values }\end{array}$ \\
\hline $\begin{array}{l}\text { main } \\
\text { semantics }\end{array}$ & set-assignment & relating \\
\hline $\begin{array}{l}\text { combined } \\
\text { semantics }\end{array}$ & none & $\begin{array}{l}\text { relating }+ \text { possible world } \\
\text { semantics }\end{array}$ \\
\hline new logics & $\begin{array}{l}\text { relatedness logics, } \\
\text { dependence logics }\end{array}$ & $\begin{array}{l}\text { the smallest logic in a given } \\
\text { relating language, e.g., } \mathbf{F} ; \\
\text { CMRL; BCL; MBCL }\end{array}$ \\
\hline
\end{tabular}

Table 2. Programme comparison

In turn, in the framework of the Torunian Programme the main tool is connection evaluation, which is a kind of generalisation of the relation that occurs between the formulae. The results obtained to date in the framework of the Torunian Programme are chiefly based on models with a single connection relation, which are a particular type of mono-relating models. We have also mentioned sample works which fall within the scope of the Torunian Programme and are concerned with connected semantics of possible worlds with relating semantics.

At this point it is useful to note that Epstein's logics consist of six systems, two systems of relatedness logic, and four systems of connection logic. ${ }^{16}$ They are all special cases of relating logic. Examples of relating logics defined based on works that fall within the scope of the Torunian Programme may include: Boolean connexive logics (BCL), modal Boolean connexive logics (MBCL), and classical mono-relating logics (CMRL). It is worth emphasising that, with regards to the Torunian Programme, the starting point for analysis is usually the smallest/basic relating logic determined in a given language through models which are

16 Apart from the logic DPC, the dependence logic also includes the logic $\mathbf{D P C} \mathbf{C}_{n}$ for an arbitrary $n>1$ (see footnote 8 ). 
not restricted by additional limitations. A typical example is the $\mathbf{F}$ logic of Jarmużek and Kaczkowski [2014].

Epstein's research on logics defined by relational models marks the beginning of the history of relating logics. Although Epstein's Programme focuses above all on the representation of content relationship and on the set-assignment semantics, there are many results and ideas within his approach that determine research directions which the Torunian Programme undertakes as a separate concern. In other words, although the Torunian Programme is an attempt to develop certain issues which have already been discussed by Epstein, there is also an intent to describe new possibilities and research directions which are not part of Epstein's Programme.

Acknowledgements. The author would like to thank Tomasz Jarmużek and Francesco Paoli for all their remarks and suggestions.

\section{References}

Carnielli, W. A., 1987, "Methods of proofs of relatedness and dependence logic", Reports on Mathematical Logic 21: 35-46.

Epstein, R. L., 1979, "Relatedness and implication", Philosophical Studies 36: 137-173. DOI: 10.1007/BF00354267

Epstein, R. L., 1987, "The algebra of dependence logic", Reports on Mathematical Logic 21: 19-34.

Epstein, R.L. (with the assistance and collaboration of: W. Carnielli, I. D'Ottaviano, S. Krajewski, R. Maddux), 1990, The Semantic Foundations of Logic. Volume 1: Propositional Logics, Springer Science+Business Media: Dordrecht. DOI: 10.1007/978-94-009-0525-2

Epstein, R. L., 2005, "Paraconsistent logics with simple semantics", Logique et Analyse 48 (189-192): 71-86.

Epstein, R. L., and S. Krajewski, 2004, "Relatedness predicate logic", Bulletin of Advanced Reasoning and Knowledge 2: 19-38.

Epstein, R. L., and D. Walton, 1979, "Preface", Philosophical Studies 36: 113114.

Fagin, R., and J. Y. Halpern, 1988, "Belief, awareness and limited reasoning", Artificial Intelligence 34: 39-76. DOI: 10.1016/0004-3702(87)90003-8 
Fariñas del Cerro, L.., and V. Lugardon, 1991, "Sequents for dependence logics", Logique et Analyse 133/134: 57-71.

Fariñas del Cerro, L., and V. Lugardon, 1997, "Quantification and dependence logics", pages 179-190 in S. Akama (ed.), Logic, Language and Computation. Applied Logic Series, Vol. 5, Springer Science+Business Media: Dordrecht. DOI: $10.1007 / 978-94-011-5638-7 \_9$

Ferguson, T. M., 2017, Meaning and Prescription in Formal Logic. Variations on the Propositional Logic of William T. Parry, Trends in Logic, Vol. 54, Springer. DOI: $10.1007 / 978-3-319-70821-8$

Iseminger, G., 1986, "Relatedness logic and entailment", The Journal of NonClassical Logic 3(1): 5-23.

Jarmużek, T., 2021, "Relating semantics as fine-grained semantics for intensional propositional logics", pages 13-30 in A. Giordani, J. Malinowski (eds.), Logic in High Definition. Trends in Logical Semantics, Springer. DOI: 10. 1007/978-3-030-53487-5_2

Jarmużek, T., and B. Kaczkowski, 2014, "On some logic with a relation imposed on formulae: tableau system F", Bulletin of the Section of Logic 43 (1/2): $53-72$.

Jarmużek, T., and M. Klonowski, 2020, "On logics of strictly-deontic modalities. A semantic and tableau approach", Logic and Logical Philosophy 29 (3): 335-380. DOI: 10.12775/LLP.2020.010

Jarmużek, T., and M. Klonowski, 2021, "Some intensional logics defined by relating semantics and tableau systems", pages 31-48 in A. Giordani, J. Malinowski (eds.), Logic in High Definition. Trends in Logical Semantics, Springer. DOI: 10.1007/978-3-030-53487-5_3

Jarmużek, T., and M. Klonowski, submitted, "Classical mono-relating logic. Theory and axiomatization".

Jarmużek, T., and J. Malinowski, 2019a, "Boolean connexive logics: semantics and tableau approach", Logic and Logical Philosophy 28 (3): 427-448. DOI: 10.12775/LLP. 2019.003

Jarmużek, T., and J. Malinowski, 2019b, "Modal Boolean connexive logics: semantics and tableau approach", Bulletin of the Section of Logic 48 (3): 213-243. DOI: $10.18778 / 0138-0680.48 .3 .05$

Klonowski, M., 2018, "A Post-style proof of completeness theorem for Symmetric Relatedness Logic S", Bulletin of the Section of Logic 47 (3): 201-214. DOI: $10.18778 / 0138-0680.47 .3 .05$ 
Klonowski, M., 2019, "Aksjomatyzacja monorelacyjnych logik wiążących" ("Axiomatization of monorelational relating logics"), $\mathrm{PhD}$ thesis, Nicolaus Copernicus University in Torun.

Klonowski, M., 2021, "Axiomatization of some basic and modal Boolean connexive logics", Logica Universalis 15: 517-536. DOI: 10.1007/s11787-02100291-4

Krajewski, S., 1982, "On relatedness logic of Richard L. Epstein", Bulletin of the Section of Logic 11 (1/2): 24-30.

Krajewski, S., 1986, "Relatedness Logic", Reports on Mathematical Logic 20: $7-14$.

Krajewski, S., 1991, "One or many logics? (Epstein's set-assignement semantics for logical calculi)", The Journal of Non-Classical Logic 8 (1): 7-33.

Ledda, A., F. Paoli and M. Pra Baldi, 2019, "Algebraic analysis of demodalised analytic implication", Journal of Philosophical Logic 48: 957-979. DOI: 10. 1007/s10992-019-09502-2

Malinowski, J., and R. Palczewski, 2021, "Relating semantics for connexive logic", pages 49-65 in A. Giordani and J. Malinowski (eds.), Logic in High Definition. Trends in Logical Semantics, Springer. DOI: 10.1007/978-3030-53487-5_4

Paoli, F., 1993, "Semantics for first degree relatedness logic", Reports on Mathematical Logic 27: 81-94.

Paoli, F., 1996, "S is constructively complete", Reports on Mathematical Logic 30: $31-47$.

Paoli, F., 2007, "Tautological entailments and their rivals", pages 153-175 in J. Y. Béziau, W. A. Carnielli, D. M. Gabbay (eds.), Handbook of Paraconsistency, College Publications: London.

Walton, D. N., 1979a, "Philosophical basis of relatedness logic", Philosophical Studies 36 (2): 115-136. DOI: 10.1007/BF00354266

Walton, D. N., 1979b, "Relatedness in intensional action chains", Philosophical Studies 36 (2): 175-223. DOI: 10.1007/BF00354268

Walton, D. N., 1982, Topical Relevance in Argumentation, John Benjamins Publishing Company: Amsterdam/Philadelphia. DOI: 10.1075/pb.iii.8

Walton, D. N., 1985, "Pragmatic inferences about actions", Synthese 65: 211233. DOI: $10.1007 / \mathrm{BF} 00869300$ 
Wansing, H., 2020, "Connexive logic", in E. N. Zalta (ed.), Stanford Encyclopedia of Philosophy, November 11, 2021. https://plato.stanford.edu/ entries/logic-connexive/

\author{
Mateusz KlOnOWsKI \\ Departament of Logic \\ Nicolaus Copernicus University in Toruń \\ Poland \\ mateusz.klonowski@umk.pl
}

